\title{
Polarization Holography in 3-Indoly-Benzylfulgimide/PMMA Film
}

\author{
Neimule Menke, ${ }^{1,2}$ Baoli Yao, ${ }^{2}$ Yingli Wang, ${ }^{2}$ and Yi Chen ${ }^{3}$ \\ ${ }^{1}$ School of Physical Science and Technology, Inner Mongolia Universtiy, Huhhot 010021, China \\ ${ }^{2}$ State Key Laboratory of Transient Optics and Photonics, Xi'an Institute of Optics and Precision Mechanics, \\ Chinese Academy of Sciences, Xi'an 710068, China \\ ${ }^{3}$ Key Laboratory of Photochemical Conversion and Optoelectronic Materials, Technical Institute of Physics and Chemistry, \\ Chinese Academy of Sciences, Beijing 100101, China
}

Correspondence should be addressed to Neimule Menke, mknml@yahoo.com.cn

Received 11 October 2010; Accepted 27 February 2011

Academic Editor: Ali Hussain Reshak

Copyright (C) 2011 Neimule Menke et al. This is an open access article distributed under the Creative Commons Attribution License, which permits unrestricted use, distribution, and reproduction in any medium, provided the original work is properly cited.

\begin{abstract}
The photochromic and photoanisotropic properties of materials can be used in ordinary and polarization holographic recording respectively. Fulgides are well known as thermally irreversible organic photochromic compounds. And it is found that there exists photoinduced anisotropy in fulgide-doped polymeric films. In this report, a 3-indoly-benzylfulgimide/PMMA film was studied as a holographic storage media. First, the spectra and dynamics of photochromic and photoanisotropic properties of the sample are measured or calculated. Second, the diffraction efficiency (DE) dynamics at $633 \mathrm{~nm}$ of four kinds of different polarization holograms recorded in this sample are measured. The maximum DE value about $1 \%$ was gotten. Third, the DE spectra and DE dynamics are theoretically calculated in detail, and a good correlation of theoretically derived DE dynamic curves and the measured experimental curves was found. From the DE spectra, it is known that at the wavelengths less than $450 \mathrm{~nm}$ or greater than $700 \mathrm{~nm}$, the nondestructive reading can be realized. The DWPS obtained in the experiments are same with the theortically deduced ones, which shows that in the orthogonal polarization holography, the polarization state of the diffracted light is orthogonal to that of the reconstruction light, which is very important to increase the SNR of the holographic storage. And all these results are applied and proved to be correct in high-density holographic image storage experiment. The area density of $2 \times 10^{8} \mathrm{bits} / \mathrm{cm}^{2}$ was obtained, and the encoded data was retrieved without error.
\end{abstract}

\section{Introduction}

Holographic optical storage becomes an important research aspect in optical storage field for its high data storage capacity and data transmission rate. Fulgides are well known as thermally irreversible organic photochromic compounds [1], which can be used as rewritable ordinary holographic recording material. But up to now, a few researches have been done on the holographic storage application of fulgide materials [2-9]. And we found that there exists photoinduced anisotropy in fulgide-doped polymeric films [10], which can be used in polarization holography application.

Here, the diffracted wave polarization states (DWPS) and the diffraction efficiencies (DE) of ordinary holograms and polarization holograms recorded in a 3-indolybenzylfulgimide/PMMA film are studied in detail. First, the photochromic and photoanisotropic properties of the sample is given, and the DE dynamics at $633 \mathrm{~nm}$ of parallel linearly, parallel circularly, orthogonal linearly, and orthogonal circularly polarization holograms, each of which read by four kinds of different polarized lights, are measured. Second, the DE formula and DWPS of parallel linearly, parallel circularly, orthogonal linearly, and orthogonal circularly polarization holograms in photochromic and photoanisotropic thin films are theoretically deduced; Third, using the formula deduced before, from the properties of the sample, the DE spectra and dynamics at $633 \mathrm{~nm}$ of four kinds of different polarization recorded holograms are theoretically calculated and compared with the experimental curves, and the DWPS obtained in the experiments are compared with the theortically deduced ones. At last, all these results have been applied in high-density holographic image storage experiment. 


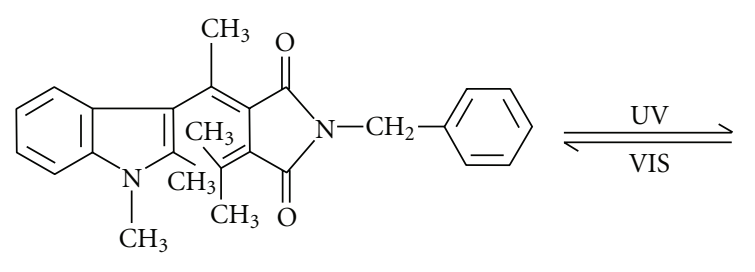

E-form

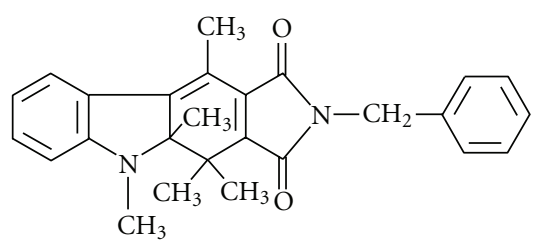

C-form

Figure 1: Molecular formula of the Indolyfulgimide and the photochromic reaction. Left, $E$-form; right, $C$-form.

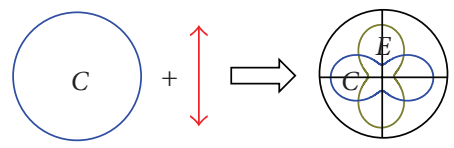

Figure 2: Angular distributions of molecules in anisotropy inducing progress.

\section{Material and Measurement Experiments}

2.1. Material. The fulgide material studied here is 3-indolybenzylfulgimide, which was synthesized by the Stobbe condensation routine [11]. The target compound of $3 \mathrm{mg}$ was dissolved in a $0.1 \mathrm{~mL} 10 \%$ (by weight) PMMA-cyclohexanone solution. Then, the solution was coated on a 1-mm thick $\mathrm{K}_{9}$ glass plane with a spin coater and dried in air. The thickness of the film is about $10 \mu \mathrm{m}$. The photochromic or photoanisotropic properties of fulgides are due to a reversible photochromic (photoisomerization) reaction that occurs between one of the colorless $E$-form (bleached state) and the $C$-form (colored state). These are the two spectrally separated photochromic forms, whose molecular formulas are shown in Figure 1.

When the colored state is irradiated by a linearly polarized $650 \mathrm{~nm}$ laser, the film returns to the bleached state and photo-induced anisotropy is produced during this process. The mechanism of photo-induced anisotropy in the fulgide film is the following [10]: anisotropic absorbing molecules of fulgide are immobilized randomly in the PMMA polymeric matrix, which shows isotropic characteristic at the initial state; when the sample is irradiated by linearly polarized light photoselection of molecules take place. Molecules with long axes parallel to the exiting light polarization direction absorb the light strongly and turn to the other form very quickly, whereas those with long axes perpendicularly orientated to exiting light polarization have low absorption and stay at the initial form. As a result, special orientation of two form molecules is induced and the sample shows optical anisotropy properties macroscopically. The angular distributions of molecules in anisotropy inducing progress are shown in Figure 2. So, under the irradiation of circularly polarized light, the sample shows also optical isotropy.

\subsubsection{Spectra of Photochromic and Photoanisotropy Properties} of the Sample. The absorption of the $C$-form $\left(A_{C}(\lambda)\right)$ and $E$-form film $\left(A_{E}(\lambda)\right)$ were measured using a UV-VIS-IR spectrophotometer (UV-3101PC, Shimadzu Inc., Japan), which were shown in Figure 3(a). And the measurement of the photo-induced dichroism is performed by measuring the transmission spectra of the film for testing light polarized parallel $\left(T_{/ /}(\lambda)\right)$ and perpendicular $\left(T_{\perp}(\lambda)\right)$ to the polarization direction of the exciting beam after the $C$-form film is excited by the linearly polarized $650 \mathrm{~nm}$ laser (shown in Figure 4(a)).

From Figures 3(a) and 4(a), the photo-induced absorption changing spectrum $\Delta A(\lambda)=A_{E}(\lambda)-A_{C}(\lambda)$ and the photo-induced dichroism spectrum $\Delta A_{D}(\lambda)=A_{\perp}(\lambda)-$ $A_{/ /}(\lambda)=\lg \left(T_{/ /}(\lambda) / T_{\perp}(\lambda)\right)$ were obtained, which are shown as solid lines in Figures 3(b) and 4(b), respectively. Assuming that $\triangle A$ and $\triangle A_{D}$ are zero outside of the band $300 \sim 800 \mathrm{~nm}$, the photo-induced refractive index changing spectrum $\Delta n(\lambda)=n_{E}(\lambda)-n_{C}(\lambda)$ and the photo-induced birefringence spectrum $\Delta n_{B}(\lambda)=n_{\perp}(\lambda)-n_{/ /}(\lambda)$ can be calculated according to the Kramers-Kronig relation [12]

$$
\begin{aligned}
\Delta n(\lambda) & =\frac{\ln 10}{2 \pi^{2} d} \text { p.v. } \int_{0}^{\infty} \frac{\Delta A\left(\lambda^{\prime}\right)}{1-\lambda^{\prime 2} / \lambda^{2}} d \lambda^{\prime}, \\
\Delta n_{B}(\lambda) & =\frac{\ln 10}{2 \pi^{2} d} \text { p.v. } \int_{0}^{\infty} \frac{\Delta A_{D}\left(\lambda^{\prime}\right)}{1-\lambda^{\prime 2} / \lambda^{2}} d \lambda^{\prime},
\end{aligned}
$$

where $n_{E}, n_{C}, n_{/ /}$, and $n_{\perp}$ are the refractive indexes of $E$ form, $C$-form, and of the film excited by linearly polarized light along the photo-induced extraordinary and ordinary axes, respectively, $d$ is the thickness of the film and p.v. denotes the Cauchy principal value of the integral. The calculated $\Delta n, \Delta n_{B}$ are plotted as dot lines in Figures 3(b) and 4(b), respectively. Kramers-Kronig relation can be satisfied during all the photochromic reaction progress, so at one wavelength $\lambda^{\prime}, \Delta n\left(\lambda^{\prime}\right)$ is proportional to $\Delta A\left(\lambda^{\prime}\right)$ and $\Delta n_{B}\left(\lambda^{\prime}\right)$ is proportional to $\Delta A_{D}\left(\lambda^{\prime}\right)$ at different exciting time. From Figures $3(\mathrm{~b})$ and $4(\mathrm{~b})$, it can be seen that at $633 \mathrm{~nm}$ in this sample, $\Delta n(633 \mathrm{~nm}) / \Delta A(633 \mathrm{~nm})=0.00994$ and $\Delta n_{B}(633 \mathrm{~nm}) / \Delta A_{D}(633 \mathrm{~nm})=0.006115$.

\subsubsection{Dynamics of Photochromic and Photoanisotropy Prop-} erties of the Sample. The transmission growing up kinetics of the sample at $633 \mathrm{~nm}$ were measured on the parallel and perpendicular directions to exciting beam polarization, when the $C$-form sample was being excited with $314 \mathrm{~mW} / \mathrm{cm}^{2}$ and $157 \mathrm{~mW} / \mathrm{cm}^{2}$ intensity linearly polarized $633 \mathrm{~nm} \mathrm{He}-$ Ne lasers $\left(I_{W}\right)$, respectively, and an $1 \mathrm{~mW} / \mathrm{cm}^{2} 633 \mathrm{~nm}$ laser beam is used as the testing beam $\left(I_{T}\right)$, the optical setup and the results are shown in Figures 5 and 6(a). From Figure 6(a), it can be seen that the photochromic reaction of 


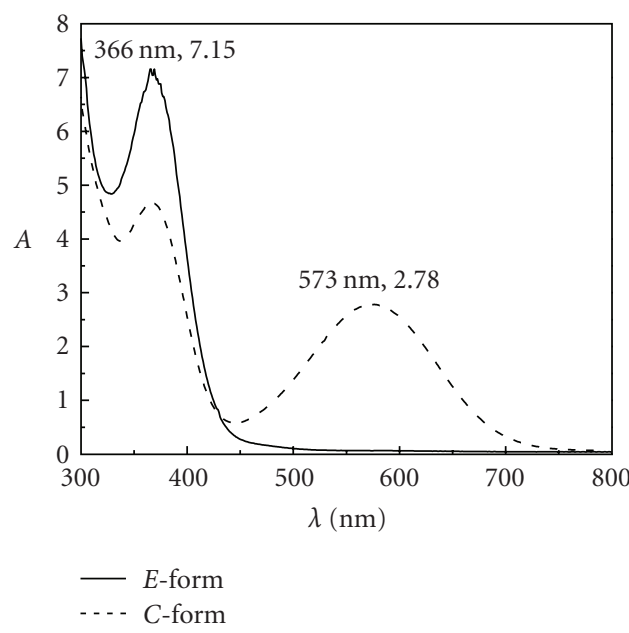

(a)

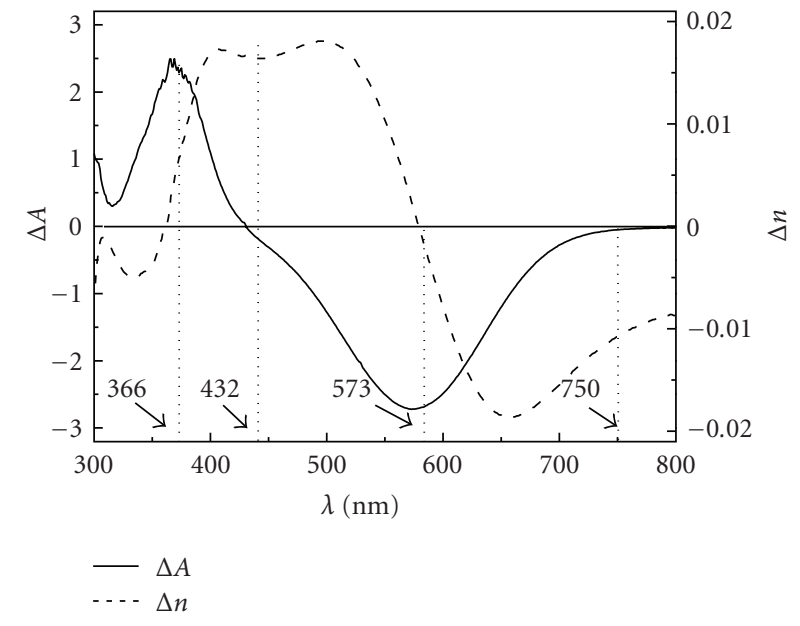

(b)

Figure 3: Spectra of photochromic properties of Indolyfulgimide/PMMA film. (a) Absorption spectra of two forms. (b) Absorption difference spectrum and the corresponding refractive index changing spectrum.

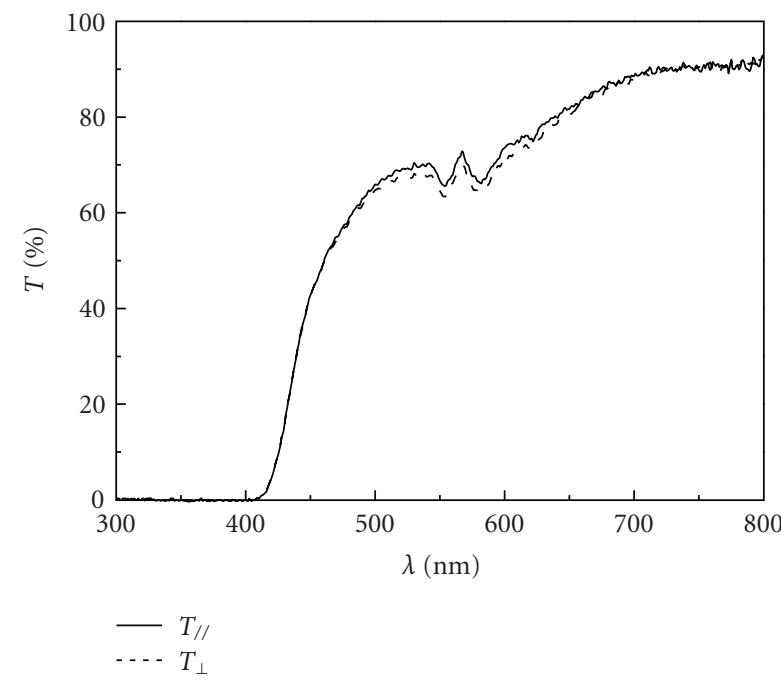

(a)

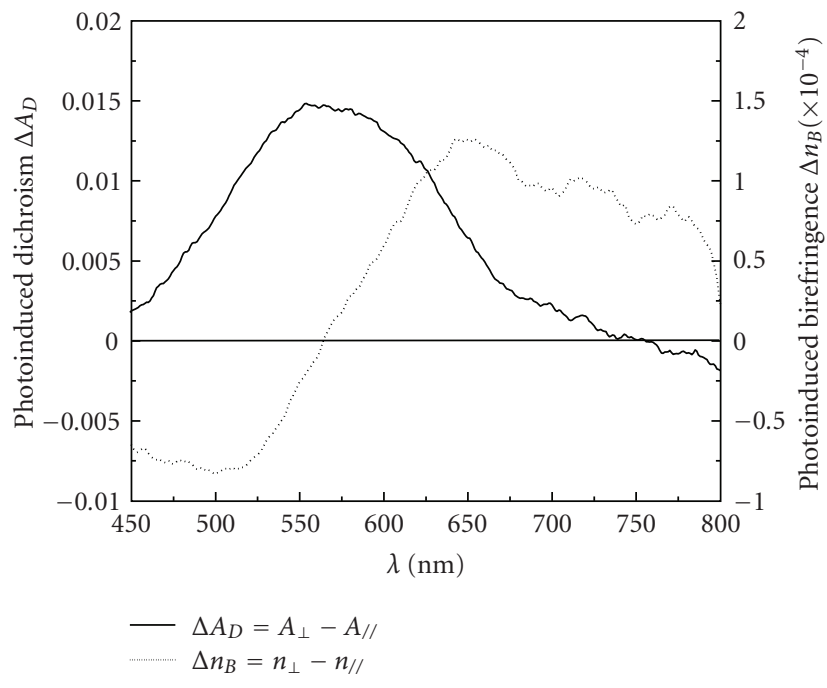

(b)

FIGURE 4: Spectra of photoanisotropic properties of Indolyfulgimide/PMMA film excited by linearly polarized light. (a) Transmission spectra on directions parallel and perpendicular to exciting beam polarization. (b) Dichroism and birefringence spectra.

fulgide material is an optical cumulating progress, which just depending on the Exposure, so it is enough to consider just one exciting beam intensity condition for the analysis.

For the analysis, we consider a fully bistable Fulgide system, neglecting side reactions like $E-Z$ isomerization or aging effects. Hence, the photochemical reaction should follow the particle number balance equations

$$
\begin{gathered}
\dot{C}=-k_{C} C+k_{E} E, \\
\dot{E}=-k_{E} E+k_{C} C, \\
F=C+E
\end{gathered}
$$

and the total concentration of fulgides molecules $F$ is constant where $E$ denotes the concentration of the $E$-isomers and $C$ the one of the $C$-isomers; dots denote derivatives with respect to time $t$ and the photoreaction rates for the annihilation of the isomers $C$ and $E$ are given by

$$
k_{C, E}=\int \gamma_{C, E}\left(\lambda_{A}\right) i_{\lambda_{A}} d \lambda_{A} .
$$

Here $\lambda_{A}$ is the actinic irradiation wavelengths, $i_{\lambda_{A}}$ is the spectral density of the light intensity, and $\gamma_{C}\left(\lambda_{A}\right)$ and $\gamma_{E}\left(\lambda_{A}\right)$ are the photochemical reaction constants of the $C$ and $E$ isomers at $\lambda_{A}$, respectively, which related to the absorption cross sections $\sigma_{C, E}$ via $\gamma_{C, E}(\lambda)=\phi_{C, E}(\lambda) \cdot \sigma_{C, E}(\lambda) / h \nu$, where $h \nu$ is the photon energy and $\phi_{C, E}$ are quantum efficiencies of the photochemical reactions. 


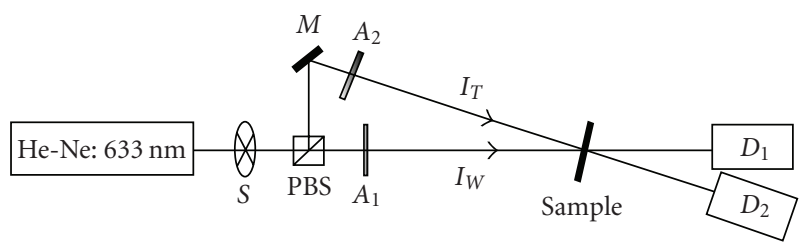

FIGURE 5: Schematic of the experimental setup for measuring transmission kinetics. PBS, polarization beam splitter; $M$, mirror; $A_{1} \sim A_{2}$, continuously adjustable attenuators; $S$, Shutter; He-Ne: $633 \mathrm{~nm}$ Helium-Neon laser; $D$, power meter; $I_{W}$, writing beam; $I_{T}$, testing beam. law

The decrease of $i_{\lambda}$ with $z$, described by Lambert-Beer's

$$
\frac{d i_{\lambda}}{d z}=-\alpha(z, t, \lambda) i_{\lambda}
$$

where $\alpha(z, t, \lambda)$ is the local absorption coefficient in the film, which equals

$$
\alpha(\lambda, z, t)=\sigma_{C}(\lambda) \cdot C(z, t)+\sigma_{E}(\lambda) \cdot E(z, t)+\alpha_{M}(\lambda, z)
$$

where $\alpha_{M}$ is the absorption of the matrix (assumed here to be photochemically inert).

Based on (2) (5), using numerical calculation method, the experimental curves were simulated (shown as the dash lines in Figure 6(b)), and the best fitting values $\gamma_{/ /}=0.00289 \mathrm{~cm}^{2} / \mathrm{mJ}, \gamma_{\perp}=0.0012 \mathrm{~cm}^{2} / \mathrm{mJ}$ were obtained. Because of the anisotropy of the molecules $\sigma_{C, E}$, the $\alpha$ and $\gamma$ values of the sample are variable with the directions at same location, here just the total average values of that of all the molecules on two special directions are obtained, which are enough for calculation of the DE kinetics, and the detail process of it will not been analyzed here. In the numerical calculations, the intensity Gaussian beam distribution of the He-Ne laser beam also has been considered, which was divided as many homogenous intensity circles. Then we can calculate the photoanisotropic transmission curves of uniformity light, which is shown in the Figure 6(c).

\subsection{Experiment Measurement of Polarization Hologram Diffraction Kinetics}

2.2.1. Measurement Setup. The system configuration for measuring the real-time hologram first-order diffraction kinetics of the Fulgide film is schematically illustrated in Figure 7. A He-Ne laser (Melles Griot Inc., USA, 25-LHP$928,632.8 \mathrm{~nm}, 35 \mathrm{~mW}$, vertical linear polarized) is used to generate recording beams (object beam $I_{O}$ and reference beam $I_{R}$ ) and readout beam (reconstruction beam $I_{C}$ ), and a laser of diode LD (Power Technology Inc., USA, IQ2A18, $405 \mathrm{~nm}, 10 \mathrm{~mW}$, vertical linear polarized) is used as the erasing light $\left(I_{E}\right)$ source. The He-Ne laser beam splitted into the $I_{O}, I_{R}$, and $I_{C}$ after beam splitter BS1 and polarization beam splitter PBS, in which $I_{R}$ and $I_{C}$ are phase conjugated (counterpropagated) beams. The diffracted light $I_{D}$ of $I_{C}$, diffracted by the dynamic holographic grating established by the interference between the $I_{O}$ and $I_{R}$, will be phase conjugated with the $I_{O}$, whose power was real time detected by a digital power meter " $D$ " (United Detector Technology company, USA, 11A Photometer/Radiometer, 254 1100 nm $I \max =10 \mathrm{~mW}$, resolution is $0.01 \mathrm{nW}$ ) and a digital oscilloscope "O" (Tektronix company, USA, TDS3032, $300 \mathrm{MHz}$, $2.5 \mathrm{GS} / \mathrm{s}, 1 \mathrm{mV})$ after reflected by the $\mathrm{BS}_{2}(\mathrm{R} 47 \%)$. The $I_{O}$ and $I_{R}$ are symmetrically incident on the sample (Fulgide film), whose intersection angle $2 \theta=16.5^{\circ}$, so the recorded grating is a nonincline grating. Shutter $S_{1}$ and $S_{2}$ controls the exposure time of red and purple beams. The continuously adjustable attenuators $A_{1} \sim A_{3}$ are used to adjust the intensities of the waves, in this experiment $I_{O}=I_{R}=$ $78.6 \mathrm{~mW} / \mathrm{cm}^{2}$ and $I_{C}=0.786 \mathrm{~mW} / \mathrm{cm}^{2}$ (i.e., $I_{O}: I_{R}: I_{C}=$ $100: 100: 1)$. This insures that the subreflection gratings formed by $I_{O}$ and $I_{C}$ as well as $I_{R}$ and $I_{C}$ can be ignored. The quarter-wave plates $Q_{1} \sim Q_{4}$ and the polarizer $P$ are used to change the polarization states of the waves; here four different polarization recording: parallel linearly polarization recording, parallel circularly polarization recording, orthogonal linearly polarization recording, and orthogonal circularly polarization recording were studied, every one was constructed by horizontal, vertical, left circular, and right circular polarized four kinds of lights like shown in Table 1.

2.2.2. Measurement Results. In four kinds of polarization recording, and different polarization reading, the diffracted wave polarization states (DWPS) obtained in the experiments are shown in Table 1 and the measured kinetic first-order diffraction efficiency (DE) curves $\eta_{+1} \sim t$ are shown in Figure 8. From them, the curves of the conditions when $I_{C}$ has same polarization state with $I_{R}$ were compared in Figure $9(\mathrm{a})$. The theoretical analysis of the results will be given in Section 3. It can be seen that there exists an optimal exposure about $2 \times 78.6 \mathrm{~mW} / \mathrm{cm}^{2} \times 3.75 \mathrm{~s} \approx$ $590 \mathrm{~mJ} / \mathrm{cm}^{2}$.

\section{Theoretical Analysis}

3.1. Background. For isotropy recording materials, only when the object light $\vec{O}$ and reference light $\vec{R}$ have components with same polarization state, there the intensity grating exists and the holograms can be recorded, called ordinary holograph. But for photo-anisotropy materials, even if the $\vec{O}$ and $\vec{R}$ have orthogonal polarization states, the holograms also can be recorded, because that at this time although the intensity of the superposed light is a constant, but its polarization state changes with the phase difference between $\vec{O}$ and $\vec{R}$. Photo-anisotropy materials can record the polarization state of exciting beam, so the holographic gratings can be recorded, called polarization holography, in which not only the intensity and phase signals of $\vec{O}$ can be stored (the photo-anisotropy is depending on the exposure), but also its polarization state can be stored. 


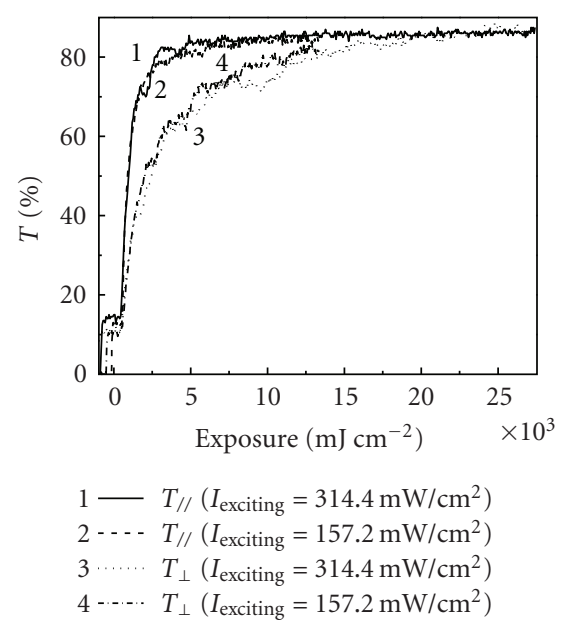

(a)

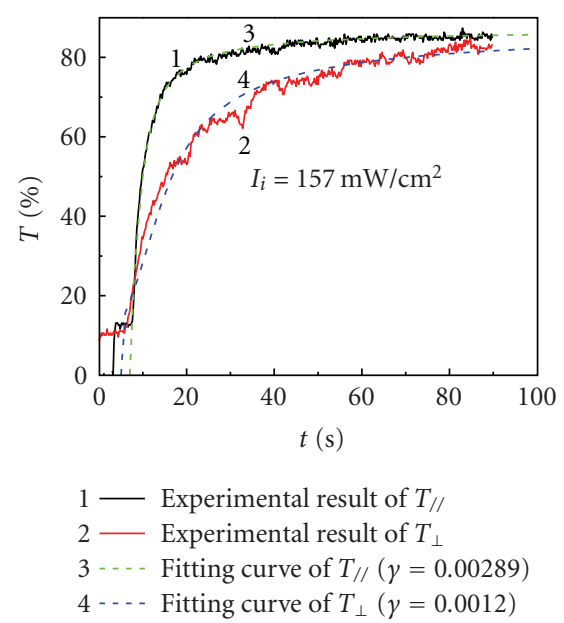

(b)

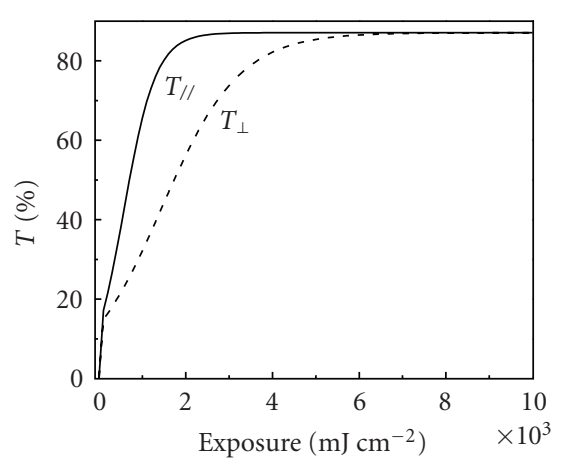

(c)

FIGURE 6: Photoanisotropic transmission curves of Indolyfulgimide/PMMA film on the directions parallel or perpendicular to exciting beam polarization depending on the exposure or erasing time. (a) Experiment curves measured at different exiting beam intensity. (b) Simulation of experimental results. (c) Calculated curves of uniformity light.

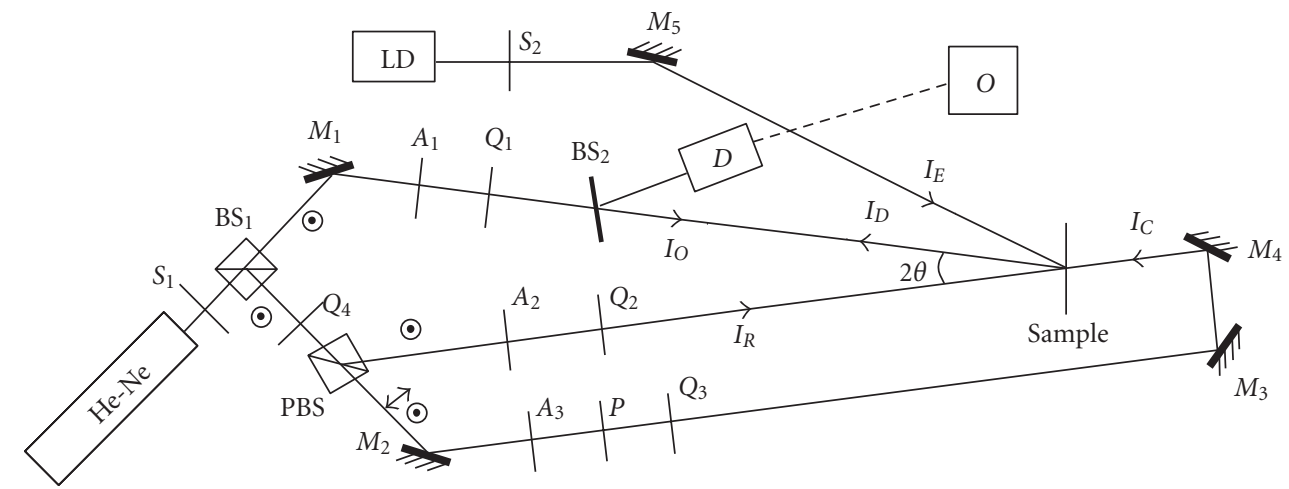

FIGURE 7: Schematic of the experimental setup for measuring real-time polarization hologram diffraction kinetics. $\mathrm{BS}_{1}$ and $\mathrm{BS}_{2}$, beam splitters; PBS, polarization beam splitter; $M_{1} \sim M_{4}$, mirrors; $A_{1} \sim A_{3}$, continuously adjustable attenuators; $P$, polarizer; $Q_{1} \sim Q_{4}$, quarterwave plates; $S_{1}$ and $S_{2}$, Shutters; $D$, digital power meter; $O$, digital oscilloscope; LD, $405 \mathrm{~nm}$ laser diode; He-Ne: $633 \mathrm{~nm}$ Helium-Neon laser; $I_{O}$, object beam; $I_{R}$, reference beam; $I_{C}$, reconstruction beam; $I_{D}$, diffracted beam; $I_{E}$, erasing violet light.

The holographic recording progress is a reaction progress between the exciting interference light and the material. So, first the properties of the exciting interference light will be analyzed and then the reaction of the sample with the light will be studied.

\subsubsection{The Properties of Exiting Interference Pattern of Object} Light and Reference Light. Choose xoz plane as incidence plane, the $\vec{O}$ incidents into the recording plane xoy with a very small incident angle $\theta$, and the $\vec{R}$ has the same incident angle (shown in Figure 10(a)), so the phase components of $\vec{O}$ and $\vec{R}$ on the $x$-axis are $\varphi_{O}=k \cdot x \cdot \sin \theta$ and $\varphi_{R}=-k \cdot x \cdot \sin \theta$, respectively, and the phase difference of them is

$$
\Delta \varphi=\varphi_{O}-\varphi_{R}=2 \cdot k \cdot x \cdot \sin \theta, \quad\left(k=\frac{2 \pi}{\lambda}\right) .
$$

(A) Parallel Polarization State. It is known that in the interference field of two coherent waves $\vec{O}$ and $\vec{R}$ with the same polarization, the polarization state is constant and same with before $\vec{E}_{1}=\vec{O}_{1}+\vec{R}_{1}=\vec{O}_{1}\left(1+\right.$ const $\left.\cdot e^{-i \Delta \varphi}\right)$, but the intensity is periodically modulated corresponding to $\Delta \varphi$ as $I_{1}=O^{2}+R^{2}+2 \cdot O \cdot R \cdot \cos \Delta \varphi$. At the condition that the amplitudes of $\vec{O}$ and $\vec{R}$ are same, that is, $O=R=A$, the interference field distribution of two special examples of it are drawn in Figures 11(a) and 11(c), including the parallel linearly polarization and parallel circularly polarization.

(B) Orthogonal Polarization State. In the interference field of two coherent waves with orthogonal polarization, that is, $\vec{R}_{2} \cdot \vec{O}_{2}{ }^{*}=\vec{O}_{2} \cdot \vec{R}_{2}{ }^{*}=0$, the intensity is constant $I_{2}=O^{2}+$ $R^{2}$, but the polarization state is periodically modulated; here two special examples of which are analyzed, including the 


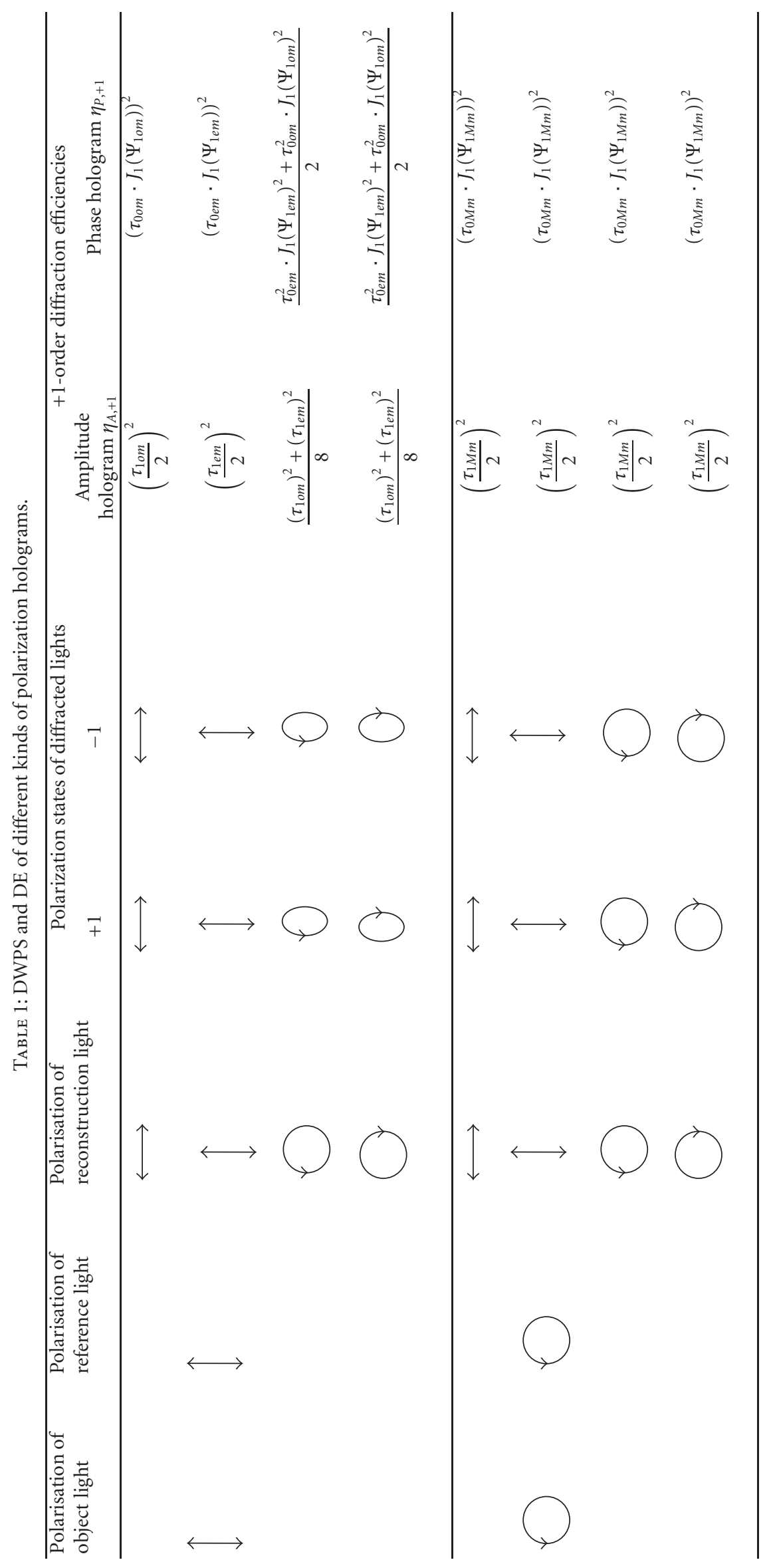




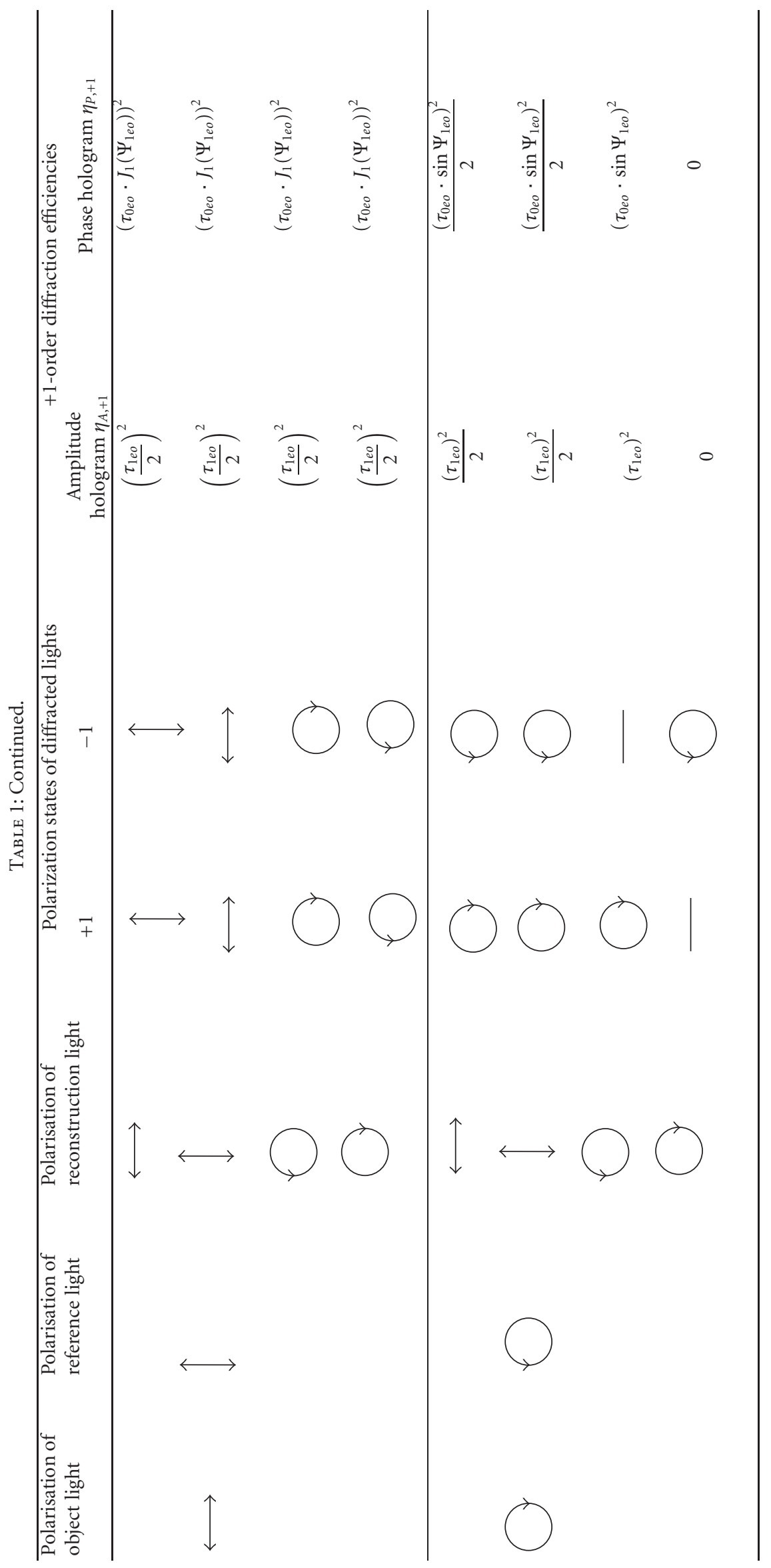




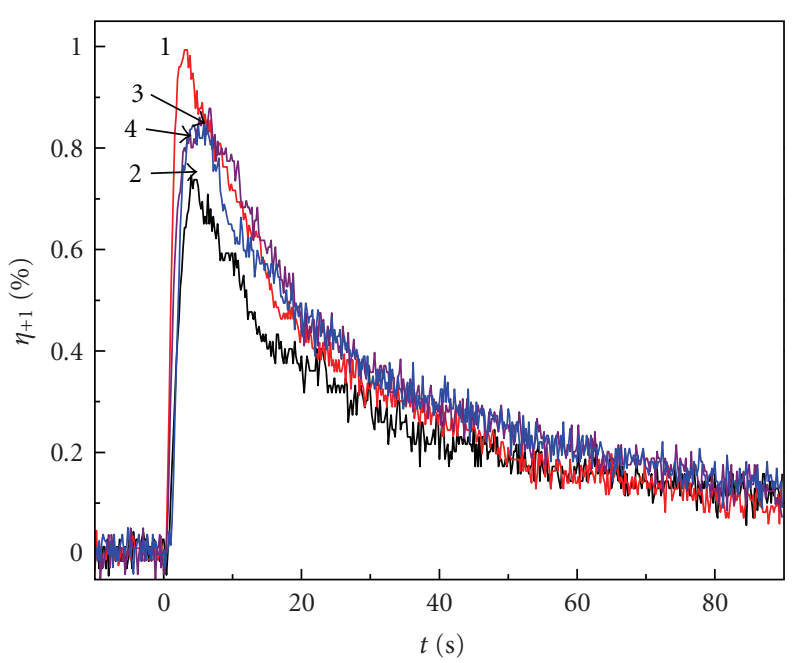

1 Vertical linearly reading $3-$ Right circularly reading 2 - Horizontal linearly reading 4 - Left circularly reading

(a)

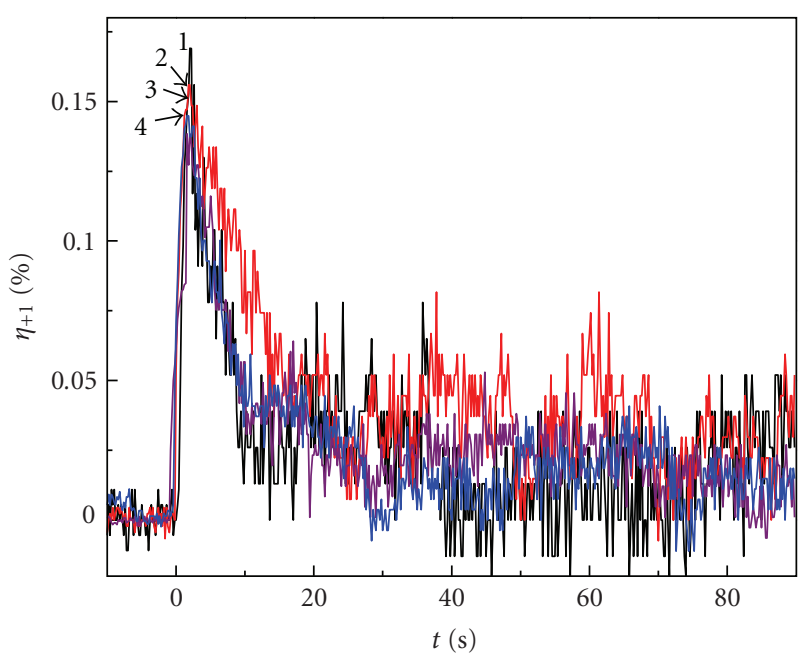

1 Vertical linearly reading $3-$ Right circularly reading 2 _ Horizontal linearly reading 4 Left circularly reading

(c)

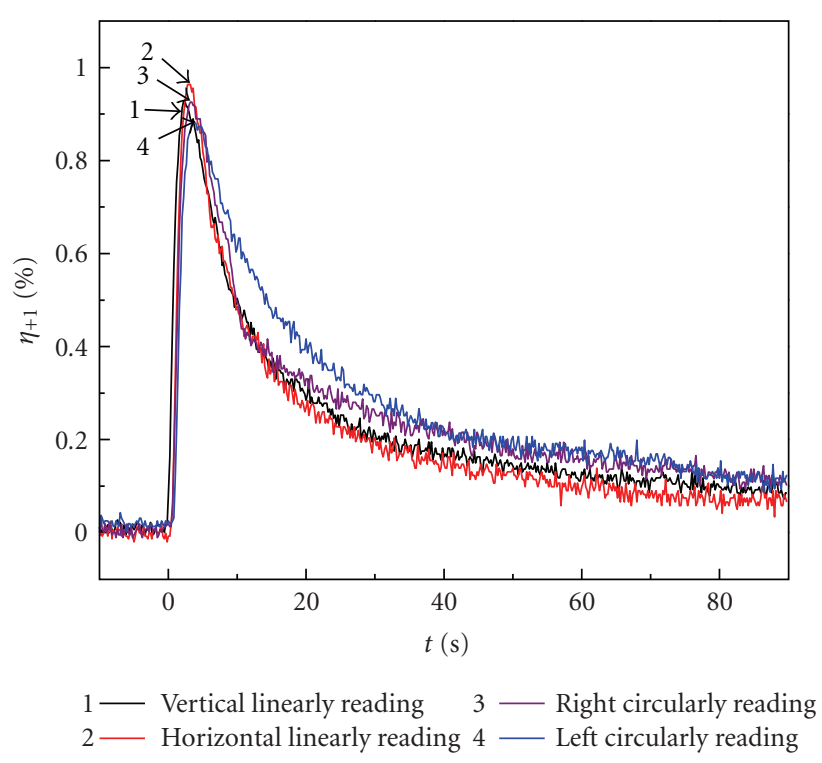

(b)

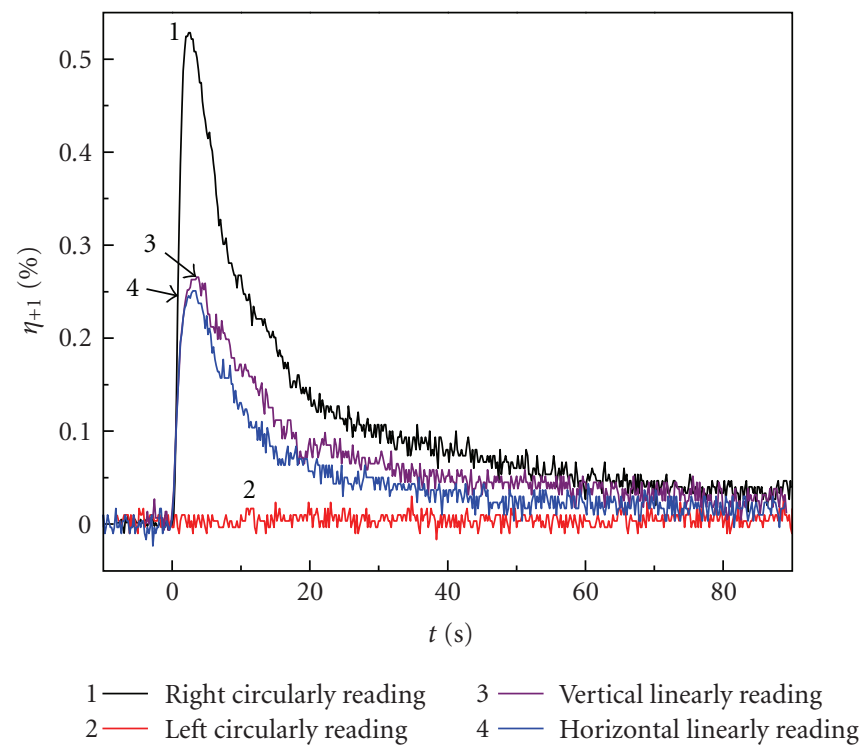

(d)

FIGURE 8: The diffraction efficiency kinetics curves comparison of different kinds of polarization recording and different kinds of reading in Fulgide film. (a) Parallel linearly polarization recording. (b) Parallel circularly polarization recording. (c) Orthogonal linearly polarization recording. (d) Orthogonal circularly polarization recording.

orthogonal linearly polarization and orthogonal circularly polarization.

(B1) Orthogonal Linearly Polarization. Suppose $\vec{O}$ and $\vec{R}$ are, respectively, horizontal and vertical linearly polarized, so:

$$
\begin{aligned}
\vec{E}_{3} & =\vec{O}_{3}+\vec{R}_{3}=O \cdot\left[\begin{array}{l}
1 \\
0
\end{array}\right] \cdot e^{i \varphi_{0}}+R \cdot\left[\begin{array}{l}
0 \\
1
\end{array}\right] \cdot e^{i \varphi_{R}} \\
& =e^{i \varphi_{O}}\left[\begin{array}{c}
O \\
R \cdot e^{-i \Delta \varphi}
\end{array}\right] \stackrel{O=R=A}{\longrightarrow} A \cdot e^{i \varphi_{O}} \cdot\left[\begin{array}{c}
1 \\
e^{-i \Delta \varphi}
\end{array}\right] .
\end{aligned}
$$

It shows that the superposed light of two orthogonal linearly polarized lights is an elliptical polarized light, whose azimuth $\alpha_{3}$ is

$$
\begin{aligned}
\alpha_{3} & =\frac{1}{2} \cdot \arctan \left[\left(\frac{2 O R}{O^{2}-R^{2}}\right) \cos \Delta \varphi\right] \\
& \stackrel{O=R=A}{=} \begin{cases}\frac{\pi}{4} & 2 k \pi-\frac{\pi}{2}<\Delta \varphi<2 k \pi+\frac{\pi}{2}, \\
-\frac{\pi}{4} & 2 k \pi-\frac{\pi}{2}<\Delta \varphi<2 k \pi+\frac{3 \pi}{2},\end{cases}
\end{aligned}
$$




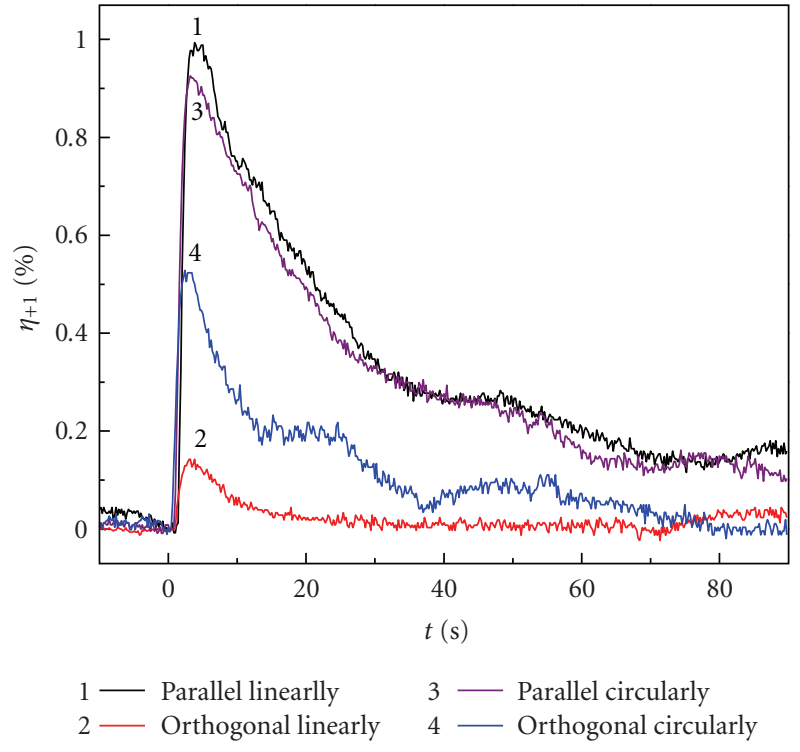

(a)

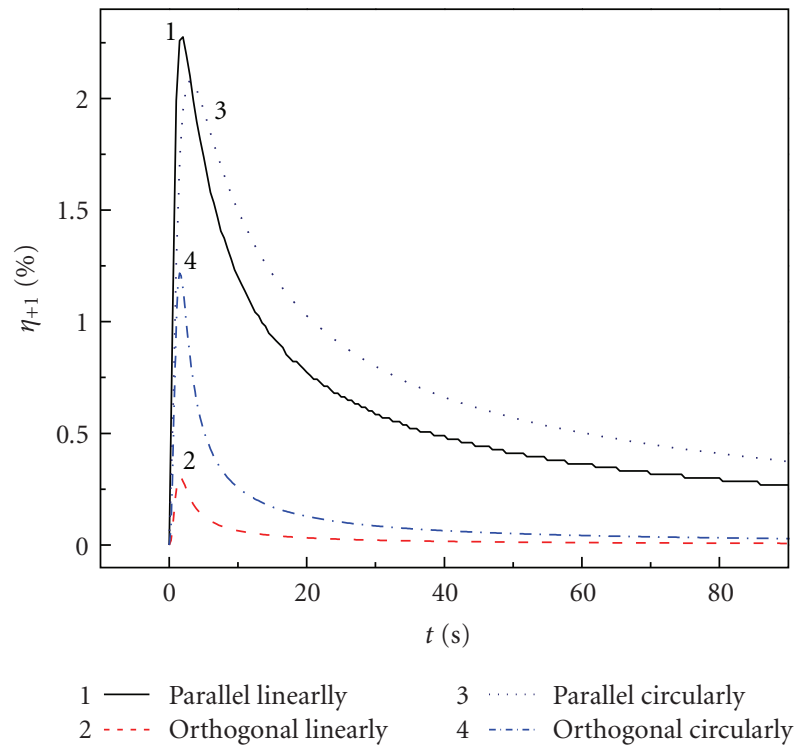

(b)

Figure 9: The diffraction efficiency kinetics curves comparison of different kinds of polarization recording holograms in Fulgide/PMMA film written by Gaussian beams. (a) Experimentally measured results. (b) Theoretically calculated results.

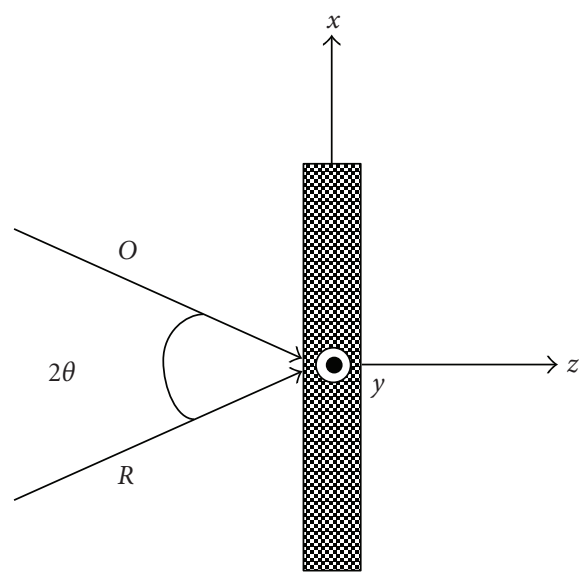

(a)

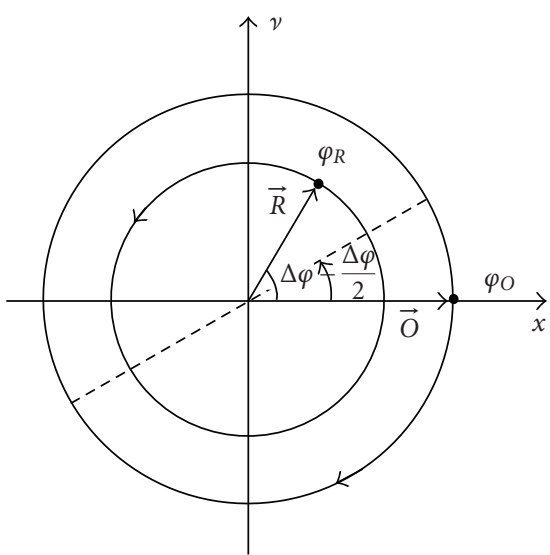

(b)

FIGURE 10: Sketch map (a) of the holographic recording setup and (b) of the superposing of two orthogonal circularly polarized lights.

and whose ellipticity $\varepsilon$ is

$$
\begin{aligned}
\mathcal{E}_{3} & =\arctan \left(\frac{L_{2}}{L_{1}}\right) \\
& =\frac{1}{2} \arcsin (\sin 2 \beta \cdot \sin \Delta \varphi) \stackrel{O=R=A}{\longrightarrow} \arctan \left(\frac{L_{2}}{L_{1}}\right)=\frac{\Delta \varphi}{2},
\end{aligned}
$$

where $L_{1}, L_{2}$ indicate the long and short axes, respectively and $\beta$ indicates the amplitude angle $(\tan \beta=R / O)$. It can be seen that the azimuth and ellipticity of interference field are periodically changing along the $\Delta \varphi$ with period $2 \pi$. The interference field distribution at the condition $O=R=A$ is drawn in Figure 11(b).
(B2) Orthogonal Circularly Polarization. Suppose $\vec{O}$ and $\vec{R}$ are, respectively, right and left circularly polarized, so:

$$
\begin{aligned}
\vec{E}_{4} & =\vec{O}_{4}+\vec{R}_{4}=O \cdot\left[\begin{array}{c}
\frac{1}{\sqrt{2}} \\
\frac{1}{\sqrt{2}} i
\end{array}\right] e^{i \varphi_{o}}+R \cdot\left[\begin{array}{c}
\frac{1}{\sqrt{2}} \\
-\frac{1}{\sqrt{2}} i
\end{array}\right] \cdot e^{i \varphi_{R}} \\
& =\frac{1}{\sqrt{2}}\left[\begin{array}{c}
O+R \cdot e^{-i \Delta \varphi} \\
\left(O-R \cdot e^{-i \Delta \varphi}\right) i
\end{array}\right] \cdot e^{i \varphi_{O}} .
\end{aligned}
$$

It is clear that the superposed light of two orthogonal circularly polarized lights is also an elliptical polarized light, whose ellipticity will not change with the $\Delta \varphi$, that is, the long and short axes are always $(O+R)$ and $(O-R)$, respectively. When $O=R=A$, the short axis is zero, that is, the 


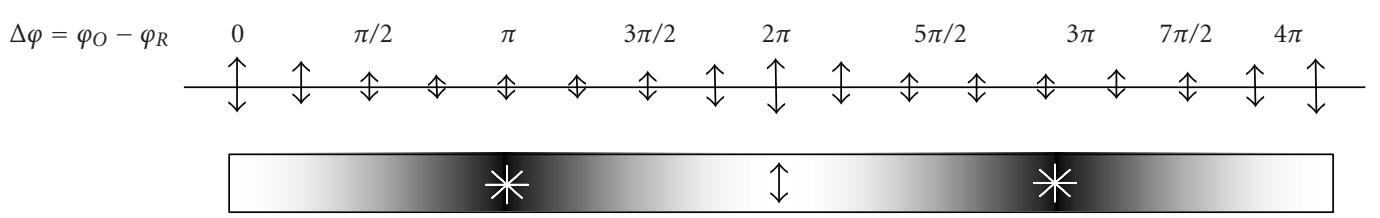

(a) Parallel linearly

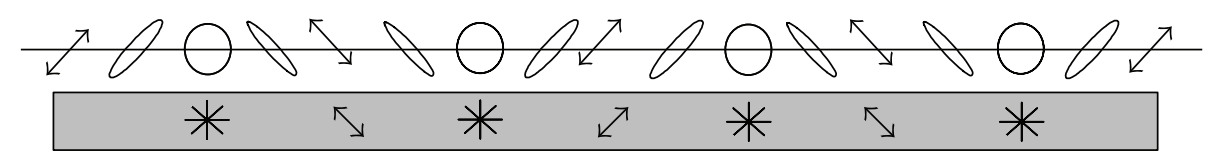

(b) Orthogonal linearly

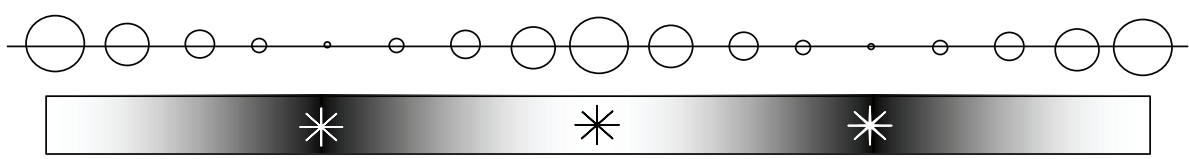

(c) Parallel circularly

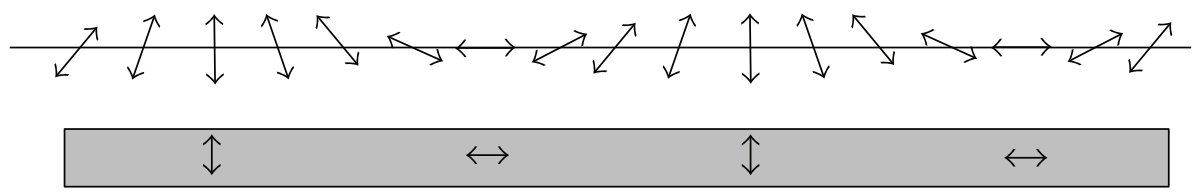

(d) Orthogonal circularly

FIGURE 11: The distributions of the interference fields in four kinds of polarization holography storage and the corresponding molecular distributions and orientations.

linearly polarized light. The azimuth of the light is $\alpha_{4}=$ $-\Delta \varphi / 2$. Figure 10 (b) is a single sketch map showing the superposing of two orthogonal circularly polarized lights. The interference field distribution at the condition $O=R=$ $A$ is drawn in Figure 11(d).

3.1.2. Analysis of the Properties of Different Kinds of Polarization Holograms and Their DWPS and DE. Under the irradiation of the interference field, the photochromic reaction will be induced in the fulgide sample, so the space modulation of the distribution of $E$-form and $C$-form molecules will be caused by the intensity modulation of optical field; photoanisotropy will be induced during the photochromic reaction under the irradiation of noncircularly polarized light, so the space modulation of the orientations (tropism) of $E$-form and $C$-form molecules will be caused by the polarization state modulation of optical field.

Here, we just consider four kinds of polarization holographic storage, including the parallel linearly, parallel circularly, orthogonal linearly, and orthogonal circularly polarized holograms. Figure 11 is a single sketch map showing the space modulation of the distributions and orientations (optical axis) of $E$-form and $C$-form molecules in these four kinds of polarization holographic storage under the linearity recording condition when $O=R=$ $A$. The arrows and ellipses on the horizontal line indicate the periodical distributions of intensity magnitudes and polarization states of the recording field corresponding to the different phase differences $\Delta \varphi$ along the $x$-axis. The rectangle frames under them indicate the distribution and orientation of the molecules under the corresponding light irradiations, where the black and white colors indicate the $C$ form and $E$-form molecules, respectively, the arrows indicate the direction of induced optical axes, and the star flower $(*)$ means the sample is isotropy at the place.

From Figure 11, it can be seen that in the parallel circularly polarized hologram just ordinary hologram exits, and in the orthogonal polarized condition, just polarization hologram exits, but in the parallel linearly polarized holography, the ordinary hologram and polarization hologram will be recorded together.

The underside, the Jones matrixes of four kinds of polarization hologram, and their DWPS and DE are analyzed under linearly recording condition. The DE are calculated using the diffraction theory of two-dimensional grating, because the thickness of the fulgide film is very small $(10 \mu \mathrm{m})$; the recorded hologram can be considered as plane hologram.

Suppose the Jones coordinate is $(x, y)$, so the Jones matrix of the hologram is

$$
T(x, y)=\left[\begin{array}{cc}
\tau_{x x} \cdot e^{i \Psi_{x x}} & \tau_{x y} \cdot e^{i \Psi_{x y}} \\
\tau_{y x} \cdot e^{i \Psi_{y x}} & \tau_{y y} \cdot e^{i \Psi_{y y}}
\end{array}\right],
$$

where $\tau$ and $\Psi$ indicate the light amplitude transmissions and light phase transmission function of the sample, and when 


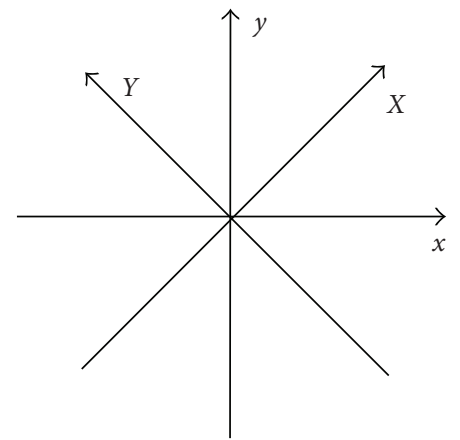

(a)

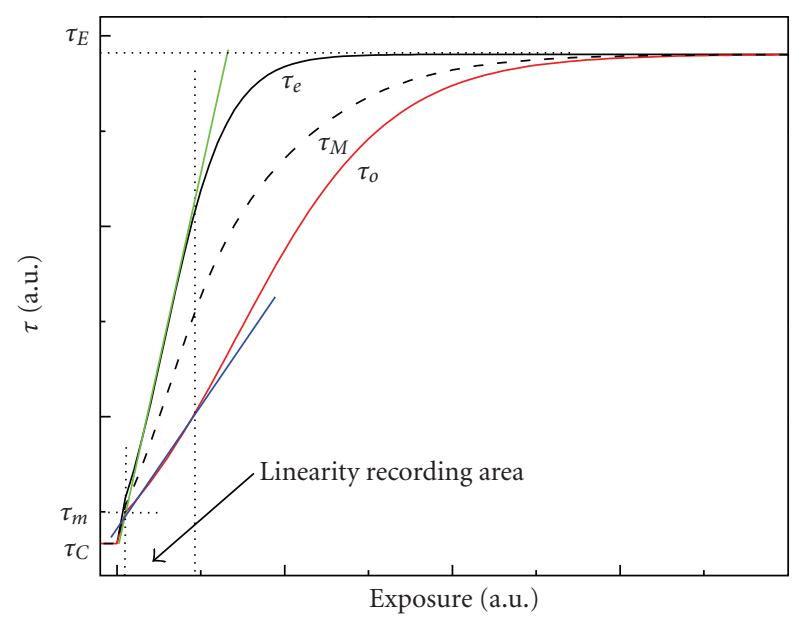

(b)

FIGURE 12: (a) The definition of coordinate system. (b) The photoinduced dichroism curve of the sample depending on the exposure.

read by the reconstruction light $\vec{C}$, the diffracted light $\vec{D}$ of the hologram will be

$$
\begin{aligned}
\vec{D}= & T(x, y) \cdot \vec{C} \\
& =\left[\begin{array}{cc}
\tau_{x x} \cdot e^{i \Psi_{x x}} & \tau_{x y} \cdot e^{i \Psi_{x y}} \\
\tau_{y x} \cdot e^{i \Psi_{y x}} & \tau_{y y} \cdot e^{i \Psi_{y y}}
\end{array}\right] \cdot\left[\begin{array}{c}
C x \\
C y
\end{array}\right] \\
& =\left[\begin{array}{c}
\tau_{x x} \cdot e^{i \Psi_{x x}} \cdot C x+\tau_{x y} \cdot e^{i \Psi_{x y}} \cdot C y \\
\tau_{y x} \cdot e^{i \Psi_{y x}} \cdot C x+\tau_{y y} \cdot e^{i \Psi_{y y}} \cdot C y
\end{array}\right] \\
& \left(\text { where } \vec{C}=\left[\begin{array}{l}
C x \\
C y
\end{array}\right]=\left[\begin{array}{l}
C x_{0} e^{i \varphi_{x}} \\
C y_{0} e^{i \varphi_{y}}
\end{array}\right]\right) .
\end{aligned}
$$

For simplify, the condition when $O=R=1$ is chosen and analysed here. And the $45^{\circ}$ contrarotated coordinate system is defined as $(X, Y)$, shown in Figure 12(a). In Figure 12(b), the photoanisotropic amplitude transmission curves are shown. The $\left(\tau_{e}-\tau_{o}\right)$ indicate the photo-induced anisotropy of the sample under the irradiation of linearly polarized light at some exposure, $\left(n_{e}-n_{o}\right)$ indicate the corresponding birefringence, $\tau_{M}=\left(\tau_{e}+\tau_{o}\right) / 2$ indicate the amplitude transmission of the sample for nonpolarized light or circularly polarized light at this time (at the area of light strips in the interference field), $\tau_{m}$ indicates the amplitude transmission of the sample before the illumination of light (at the area of dark strips in the interference field, isotropy), $\tau_{E}$ and $\tau_{C}$ indicate the amplitude transmission of the $E$-form and $C$-form sample, respectively, $n_{M}, n_{m}, n_{E}$, and $n_{C}$ indicate the corresponding refractive index of the sample, $\Psi_{i}=k_{0} \cdot n_{i}$. $d(i=e, o, M, m, E, C)$, and it is defined that $\tau_{0 i j}=\left(\tau_{i}+\tau_{j}\right) / 2$, $\tau_{1 i j}=\left(\tau_{i}-\tau_{j}\right) / 2, \Psi_{0 i j}=\left(\Psi_{i}+\Psi_{j}\right) / 2, \Psi_{1 i j}=\left(\Psi_{i}-\Psi_{j}\right) / 2(i, j=$ $e, o, M, m, E, C)$. In the Figure 12(b), the linearity recording area is given, that is, the linearity recording area of the increasing curve of $\tau_{e}$, where it is insured that the light amplitude transmission changing of the sample has the direct ratio with the exposure no matter using what kind of polarization holographic recording and reading.

(1) Parallel Linearly Polarization Recording. From Figure 11, it can be seen that the photo-induced optical axis is along the $y$-axis, so

$$
T_{1}=\left[\begin{array}{cc}
\tau_{x} \cdot e^{i \Psi_{X}} & 0 \\
0 & \tau_{y} \cdot e^{i \Psi_{Y}}
\end{array}\right]
$$

Under the linearity recording condition,

$$
\begin{aligned}
\tau_{x} & =\tau_{0 o m}+\tau_{1 o m} \cdot \cos \Delta \varphi, \\
\tau_{y} & =\tau_{0 e m}+\tau_{1 e m} \cdot \cos \Delta \varphi, \\
\Psi_{x} & =\Psi_{0 o m}+\Psi_{1 o m} \cos \Delta \varphi, \\
\Psi_{y} & =\Psi_{0 e m}+\Psi_{1 e m} \cos \Delta \varphi .
\end{aligned}
$$

For the amplitude recording material, $\Psi_{x}=\Psi_{y}=\Psi_{0}$. If the hologram is reconstructed with $\vec{C}$, which has same incident angle with $\vec{R}$, using (12) the $\vec{D}$ can be calculated as

$$
\begin{aligned}
\vec{D}_{A 1}= & T_{A 1} \cdot \vec{C} \\
= & e^{i \Psi_{0}} \cdot\left[\begin{array}{cc}
\tau_{0 o m}+\tau_{1 o m} \cos \Delta \varphi & 0 \\
0 & \tau_{0 e m}+\tau_{1 e m} \cdot \cos \Delta \varphi
\end{array}\right] \\
& \cdot\left[\begin{array}{c}
C x \\
C y
\end{array}\right] \cdot e^{-i(\Delta \varphi / 2)} \\
= & e^{i \Psi_{0}} \cdot\left[\begin{array}{l}
\tau_{0 o m} \cdot C x \\
\tau_{0 e m} \cdot C y
\end{array}\right] \cdot e^{-i(\Delta \varphi / 2)}+e^{i \Psi_{0}} \cdot \frac{1}{2}\left[\begin{array}{l}
\tau_{10 m} \cdot C x \\
\tau_{1 e m} \cdot C y
\end{array}\right] \\
& \cdot e^{-i(3 \Delta \varphi / 2)}+e^{i \Psi_{0}} \cdot \frac{1}{2}\left[\begin{array}{l}
\tau_{1 o m} \\
\tau_{1 e m} \cdot C x
\end{array}\right] \cdot e^{i(\Delta \varphi / 2)} .
\end{aligned}
$$

So, the first-order DE of it would be

$$
\begin{aligned}
\eta_{A, \pm 1} & =\frac{1}{4} \cdot \frac{\left(\tau_{1 o m} \cdot|C x|\right)^{2}+\left(\tau_{1 e m} \cdot|C y|\right)^{2}}{|C x|^{2}+|C y|^{2}} \\
& =\frac{1}{4} \cdot\left(\tau_{1 o m}{ }^{2} \cdot \frac{|C x|^{2}}{|C x|^{2}+|C y|^{2}}+\tau_{1 e m}{ }^{2} \cdot \frac{|C y|^{2}}{|C x|^{2}+|C y|^{2}}\right) .
\end{aligned}
$$


If $\vec{R}$ (vertical linearly polarized) itself is used as $\vec{C}$, the $\eta_{ \pm 1}=\left(\tau_{1 e m} / 2\right)^{2}$ and the DWPS are the same as $\vec{C}$ (shown in Table 1). If a horizontal polarized light is used as $\vec{C}$, the $\eta_{ \pm 1}=\left(\tau_{\text {lom }} / 2\right)^{2}$ and the DWPS are the same as $\vec{C}$ (shown in Table 1). But at other stations the diffracted lights are the superposed light of the diffracted lights of vertical and horizontal polarized light components of $\vec{C}$; when reconstruct with circularly polarized light, the diffracted light will be ellipse polarized, whose long axes are along the vertical direction, and DE is the average value of that of horizontal and vertical polarized lights. For the phase recording material, $\tau_{x}=\tau_{y}=\tau_{0}$, using the same method the DWPS and DE of it can be deduced, in which four typical ones are written in Table 1.

At the nonlinearly recording condition, the grating of the amplitude and refractive index will no longer be a single sine function distributing, but will be a superposition of a lot of different sine function distribution

$$
\begin{gathered}
\tau_{i}=\tau_{0 i}+\sum_{n=1}^{\infty} \tau_{n i} \cdot \cos (n \cdot \Delta \varphi), \quad(i=x, y), \\
\psi_{i}=\psi_{0 i}+\sum_{n=1}^{\infty} \psi_{n i} \cdot \cos (n \cdot \Delta \varphi), \quad(i=x, y) .
\end{gathered}
$$

So when reconstruct with horizontal or vertical polarized light, the polarization states of the diffracted lights will not change, and the \pm 1 -order diffraction efficiencies of the amplitude grating and phase grating will be, respectively, $\eta_{A, \pm 1}=\tau_{1 i}{ }^{2} / 4$ and $\eta_{P, \pm 1}=\left(\tau_{0 i} \cdot J_{1}\left(\Psi_{1 i}\right)\right)^{2}(i=x, y)$. But when reconstruct with circularly polarized light, the diffracted lights are the superposed light of the diffracted lights of horizontal and vertical polarized light component of the reconstruction beam, so it will be ellipse polarized lights, whose long axes are along the vertical direction, and the diffraction efficiencies are the their average value.

(2) Parallel Circularly Polarization Recording. From Figure 11, it can be seen that the sample is isotropy at this condition, so

$$
T_{2}=\left[\begin{array}{cc}
\tau \cdot e^{i \Psi} & 0 \\
0 & \tau \cdot e^{i \Psi}
\end{array}\right] .
$$

Under the linearity recording condition,

$$
\begin{aligned}
& \tau=\tau_{0 M m}+\tau_{1 M m} \cdot \cos \Delta \varphi, \\
& \Psi=\Psi_{0 M m}+\Psi_{1 M m} \cos \Delta \varphi .
\end{aligned}
$$

Using (12), the first-order DWPS and DE can be calculated when it is read by bragg angle entered arbitrary polarized light, in which the four typical ones were written in Table 1. At the nonlinearly recording condition, the same as the parallel linearly polarization holography, the grating will no longer be a single sine function distributing. But the sample is isotropy, that is, $T x=T y$. So the polarization state of the diffracted lights are always the same as the $\vec{C}$, and the diffraction efficiency is invariable with the polarization state of the $\vec{C}, \eta_{A, \pm 1}=\tau_{1}^{2} / 4, \eta_{P, \pm 1}=\left(\tau_{0} \cdot J_{1}\left(\Psi_{1}\right)\right)^{2}$.
(3) Orthogonal Linearly Polarization Recording. For the orthogonal linearly polarization hologram, the superposed light is elliptically polarized light, whose long axes have $\alpha=$ $\pm \pi / 4$ angle between the $x$-axis, that is, along the $X, Y$ axes. So first the Jones vector of the interference field Equation (7) is turned into $X, Y$ coordinate

$$
\begin{aligned}
\left.\vec{E}_{3}\right|_{O=R=A} & =A \cdot e^{i \varphi_{O}} \cdot\left[\begin{array}{cc}
\cos \alpha & \sin \alpha \\
-\sin \alpha & \cos \alpha
\end{array}\right] \cdot\left[\begin{array}{c}
1 \\
e^{-i \Delta \varphi}
\end{array}\right] \\
& \stackrel{\alpha=\pi / 4}{=} \sqrt{2} A \cdot e^{i \varphi_{O}} \cdot\left[\begin{array}{c}
\cos \frac{\Delta \varphi}{2} \\
i \cdot \sin \frac{\Delta \varphi}{2}
\end{array}\right] .
\end{aligned}
$$

The optical anisotropy induced by elliptically polarized light should have direct ratio with the intensity difference between $X, Y$ two directions $I_{3 X}-I_{3 Y}=2 A^{2} \cos \Delta \varphi$. So, the amplitude transmission Jones matrix of the holograph can be written as

$$
T_{3}(X, Y)=\left[\begin{array}{cc}
\tau_{X} \cdot e^{i \Psi_{X}} & 0 \\
0 & \tau_{Y} \cdot e^{i \Psi_{Y}}
\end{array}\right],
$$

where

$$
\begin{aligned}
\tau_{X} & =\tau_{0 e o}+\tau_{1 e o} \cdot \cos \Delta \varphi, \\
\tau_{Y} & =\tau_{0 e o}-\tau_{1 e o} \cdot \cos \Delta \varphi, \\
\Psi_{X} & =\Psi_{0 e o}+\Psi_{1 e o} \cdot \cos \Delta \varphi, \\
\Psi_{Y} & =\Psi_{0 e o}-\Psi_{1 e o} \cdot \cos \Delta \varphi .
\end{aligned}
$$

And then the Jones matrix of the holograph is turned into usually used $x, y$ coordinate:

$$
\begin{aligned}
& T_{3}(x, y)= {\left[\begin{array}{cc}
\cos \alpha & -\sin \alpha \\
\sin \alpha & \cos \alpha
\end{array}\right] \cdot\left[\begin{array}{cc}
\tau_{X} \cdot e^{i \Psi_{X}} & 0 \\
0 & \tau_{Y} \cdot e^{i \Psi_{Y}}
\end{array}\right] } \\
& \cdot\left[\begin{array}{cc}
\cos \alpha & \sin \alpha \\
-\sin \alpha & \cos \alpha
\end{array}\right] \\
& \stackrel{\alpha=\pi / 4}{=} \frac{1}{2}\left[\begin{array}{cc}
\tau_{X} \cdot e^{i \Psi_{X}}+\tau_{Y} \cdot e^{i \Psi_{Y}} & \tau_{X} \cdot e^{i \Psi_{X}}-\tau_{Y} \cdot e^{i \Psi_{Y}} \\
\tau_{X} \cdot e^{i \Psi_{X}}-\tau_{Y} \cdot e^{i \Psi_{Y}} & \tau_{X} \cdot e^{i \Psi_{X}}+\tau_{Y} \cdot e^{i \Psi_{Y}}
\end{array}\right] .
\end{aligned}
$$

Using (12), the first-order DWPS and DE can be calculated when it is read by bragg angle entered lights, in which the four typical ones were written in Table 1. It can be deduced that the $x$ and $y$ components of the \pm 1 -order diffracted lights are reversed comparing to the $\vec{C}$, so their long axes are symmetry distributed on the $X$-axis with the long axis of $\vec{C}$, where four special conditions exit: When the $\vec{C}$ is vertical, horizontal, right circularly or left circularly polarized light, the polarization state of the diffracted light is orthogonal to that of $\vec{C}$. The polarization of the \pm 2 order diffracted lights are same with the reconstruction light. The \pm 1 -order DE 
of amplitude and phase holograms are respectively $\left(\tau_{1 e o} / 2\right)^{2}$ and $\left(\tau_{0 e o} \cdot J_{1}\left(\Psi_{1 e o}\right)\right)^{2}$.

At the nonlinearly recording condition, $\left(\tau_{e}-\tau_{o}\right)$ and $\left(n_{e}-n_{o}\right)$ will not have direct ration with the exposure, but the anisotropy distribution will not change, so the diffraction efficiency equations at the linearly recording condition also can be used.

(4) Orthogonal Circularly Polarization Recording. From Section 3.1.1, it can be seen that the superposed light field of two orthogonal circularly polarized lights (the same intensity) is a linearly polarized light, and the intensity will not change with $\Delta \varphi$, so every where in the sample its amplitude transmission matrix in the coordinate of the its main axes should be

$$
T_{4}(e, o)=\left[\begin{array}{cc}
\tau_{e} \cdot e^{i \Psi_{e}} & 0 \\
0 & \tau_{o} \cdot e^{i \Psi_{o}}
\end{array}\right]
$$

The optical axis of the superposed light is changing with $\Delta \varphi$, which has $\Delta \varphi / 2$ angle with the $X$-axis to counterclockwise direction, so the angle with the $x$-axis is $\alpha=$ $\Delta \varphi / 2+\pi / 4$. So, the Jones matrix of the recording material in the $x, y$ coordinate will be

$$
T_{4}(x, y)=\left[\begin{array}{ll}
\tau_{e} \sin ^{2} \frac{\Delta \varphi}{2} \cdot e^{i \Psi e}+\tau_{o} \cdot \cos ^{2} \frac{\Delta \varphi}{2} \cdot e^{i \Psi o} & -\frac{1}{2} \cdot \sin \Delta \varphi \cdot\left(-\tau_{e} \cdot e^{i \Psi e}+\tau_{o} \cdot e^{i \Psi o}\right) \\
-\frac{1}{2} \cdot \sin \Delta \varphi \cdot\left(-\tau_{e} \cdot e^{i \Psi e}+\tau_{o} \cdot e^{i \Psi o}\right) & \tau_{e} \cos ^{2} \frac{\Delta \varphi}{2} \cdot e^{i \Psi e}+\tau_{o} \cdot \sin ^{2} \frac{\Delta \varphi}{2} \cdot e^{i \Psi o}
\end{array}\right]
$$

Using (12), it can be deduced that, for the orthogonal circularly polarization holographic recording, there just exist the 0 -order and \pm 1 -order diffracted lights. The polarization state of the 0 -order diffracted light is same with that of $\vec{C}$, and the polarization state of the +1 and -1 -order diffracted lights are always right and left circularly polarized, respectively, which are same with that of $\vec{O}$ and $\vec{R}$. When the $\vec{C}$ is an arbitrary polarized light, it has the 0 -order and \pm 1 -order diffracted lights, whose left circularly polarized light component will diffract the +1 -order light, and the right circularly polarized light component will diffract the -1-order light. The \pm 1 -order DE of amplitude holograph are respectively $\eta_{A,+1}=$ $\left(\tau_{1 e o} \cdot C_{l 0} / C_{0}\right)^{2}$ and $\eta_{A,-1}=\left(\tau_{1 e o} \cdot C_{r 0} / C_{0}\right)^{2}$, that of the phase holograph are, respectively $\eta_{P,+1}=\left(\tau_{0 e o} \cdot \sin \Psi_{1 e o} \cdot C_{l 0} / C_{0}\right)^{2}$ and $\eta_{P,-1}=\left(\tau_{0 e o} \cdot \sin \Psi_{1 e o} \cdot C_{r 0} / C_{0}\right)^{2}$, where the $C_{0}, C_{l 0}, C_{r 0}$ are the amplitudes of $\vec{C}$ and of its left and right circularly polarized light components. In which, when the $\vec{C}$ is left circularly polarized light (same with $\vec{R}$ ), only the 0 -order and +1 order diffracted lights exist, and the polarization state of the +1 order diffracted light is orthogonal to that of $\vec{C}$, and $\eta_{A,+1}=\left(\tau_{1 e o}\right)^{2}, \eta_{A,-1}=0, \eta_{P,+1}=\left(\tau_{0 e o} \cdot \sin \Psi_{1 e o}\right)^{2}$ and $\eta_{P,-1}=0$. When the $\vec{C}$ is right circularly polarized light (orthogonal with $\vec{R}$ ), only the 0 -order and -1 order diffracted lights exist, and the polarization state of the -1order diffracted light is also orthogonal to that of $\vec{C}$, and $\eta_{A,+1}=0, \eta_{A,-1}=\left(\tau_{1 e o}\right)^{2}, \eta_{P,+1}=0$ and $\eta_{P,-1}=$ $\left(\tau_{0} \cdot \sin \Psi_{1 e o}\right)^{2}$, which are written in Table 1. At the nonlinearly recording condition, the same as the orthogonal linearly polarization holographic recording, the anisotropy grating distribution will not change, so the diffraction efficiency equations at the linearly recording condition also can be used.

3.1.3. DWPS and DE Formulas of Different Kinds of Hologram. In Table 1, the DWPS and DE of parallel linearly, parallel circularly, orthogonal linearly and orthogonal circularly polarization holographs at the linearly, recording condition are summarized, when reconstructed with linearly polarized lights and circularly polarized lights. Usually the holograms are mixed hologram, whose DE approximately equals to the summation of that of the amplitude grating and phase grating: $\eta_{1}=\eta_{a 1}+\eta_{p 1}$.

It can be seen that the DWPS obtained in the experiments are the same as the theortically deduced ones. And in the orthogonal polarization holography, if reconstructed with the reference light, the polarization state of the diffracted light is orthogonal to that of the reconstruction light, which is very important to increase the SNR of the holographic storage. Because that the polarization state of the scattered noise light is almost the same as that of the reconstruction light, which can be filtered by using the polarizer and quarter-wave plate.

3.2. Theoretical Calculations of DE of Fulgide Film. In this section, the DE spectra and DE dynamic curves at $633 \mathrm{~nm}$ of different kinds of holograms recorded in the sample will be calculated from the properties of the sample written in Section 2, by using the theory written in Section 3.1. In Section 2, it is given that for fulgide materials the photochromic and photo-induced anisotropy phenomenon are optical accumulating progresses, so the holographic recording progress is also an accumulating progress, which just depending on the exposure (average exposure $\bar{E}=2$. $I_{0} \cdot t$ ), so it is enough to consider just one exciting beam intensity condition. For the simplicity, the object refractive ratio (ORR) is chosen as $1: 1$, that is, $O=R=A=\left(I_{0}\right)^{1 / 2}$.

\subsubsection{Theoretical Calculation of $D E(633 \mathrm{~nm}) \sim \bar{E}$ Curves on the Exposure of Uniformity Beams}

(1) The Calculation of DE in the Parallel Polarization Recording Condition. For parallel polarization recording condition, the optical intensity distribution should be

$I(\Delta \varphi)=O^{2}+R^{2}+2 \cdot O \cdot R \cdot \cos \Delta \varphi=2 \cdot I_{0} \cdot(1+\cos \Delta \varphi)$. 
So at recording time $t$, the exposure $(E)$ distribution on the sample is

$$
E(\Delta \varphi, t)=I(\Delta \varphi) \cdot t=2 \cdot I_{0} \cdot(1+\cos \Delta \varphi) \cdot t .
$$

So, for different kinds of $\vec{C}$, the $T(\vec{C}, \Delta \varphi, t) \sim \Delta \varphi$ of the parallel linearly and parallel circularly recorded holograms at different $t$ in the sample can be obtained from $T \sim E$ curves shown in Figure 6(c)

$$
\begin{aligned}
T_{(\Delta \varphi, t)}= & T_{(E(\Delta \varphi))} \\
= & \left\{\begin{array}{c}
T_{/ /\left(2 \cdot I_{0} \cdot(1+\cos \Delta \varphi) \cdot t\right)} \\
\text { parallel linearly recording, } \vec{O} / / \vec{R} / / \vec{C} \\
T_{\perp\left(2 \cdot I_{0} \cdot(1+\cos \Delta \varphi) \cdot t\right)} \\
\text { parallel linearly recording, } \vec{O} / / \vec{R} \perp \vec{C} \\
\frac{\left(T_{/ /}+T_{\perp}\right)_{\left(2 \cdot I_{0} \cdot(1+\cos \Delta \varphi) \cdot t\right)}^{2}}{\text { parallel circularly recording. }}
\end{array}\right.
\end{aligned}
$$

(a) The Calculation of DE of the Amplitude Gratings $\left(\eta_{A}\right)$. The amplitude transmission changing curves on the phase difference $\tau \sim \Delta \varphi$ at $t$ will be

$$
\tau(\Delta \varphi, t)=[T(\Delta \varphi, t)]^{1 / 2} .
$$

Every $\tau \sim \Delta \varphi$ grating curves at different $t$ can be spread into Thaler progression, that is, simulated with the formula

$$
\tau=\tau_{0}+\sum_{n=1}^{10} \tau_{n} \cdot \cos (n \cdot \Delta \varphi)
$$

Such the different order amplitude transmission gratings' amplitude values $\tau_{n}(n=0,1, \ldots, 10)$ at different $t$ can be obtained. So the changing curves of $\tau_{n}(n=0,1, \ldots, 10)$ on the recording exposure $\tau_{n} \sim \bar{E}$ can be obtained, and then the different order DE changing curves of amplitude gratings on the recording exposure $\left(\eta_{A, \pm n} \sim \bar{E}\right)$ can be calculated as

$$
\eta_{0}=\tau_{0}^{2}, \quad \eta_{A, \pm n}=\frac{\tau_{n}^{2}}{4}, \quad(n=1,2, \ldots, 10)
$$

(b) The Calculation of DE of the Phase Gratings $\left(\eta_{P}\right)$. From the $T(t) \sim \Delta \varphi$, the $A(t) \sim \Delta \varphi$ curves can be calculated

$$
A(\Delta \varphi, t)=\log \left(\frac{1}{T(\Delta \varphi, t)}\right) .
$$

And then every $A \sim \Delta \varphi$ grating curves at different $t$ can be spread into Thaler progression, that is, simulated with the underside formula

$$
A=A_{0}+\sum_{i=1}^{10} A_{i} \cdot \cos (i \cdot \Delta \varphi)
$$

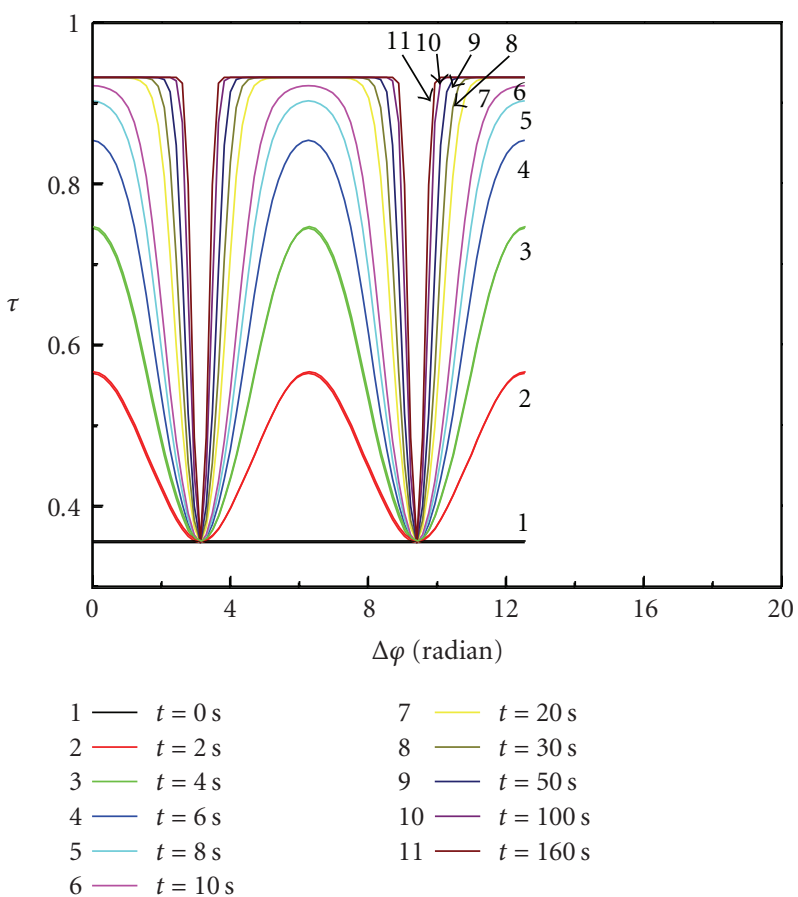

FIGURE 13: The amplitude transmission changing curves depending on the phase difference on the direction of the exiting beam polarization at different recording time.

So, the different order absorption gratings' amplitude values $A_{i}(i=0,1, \ldots, 10)$ for different kinds of $\vec{R}$ at different $t$ can be obtained. From Section 2, it is known that, at one wavelength, between the $\Delta A$ and $\Delta n$ exit direct ratio relationship. And then after multiplexed with responding ratio value, the different order phase gratings' amplitude values $n_{i}(i=0,1, \ldots, 10)$ for different polarization $\vec{R}$ at different $t$ can be got

$$
n_{i}=\text { const }_{i} \cdot A_{i} \text {. }
$$

So, the different order DE changing curves of phase gratings on recording exposure $\left(\eta_{A, \pm n} \sim \bar{E}\right)$ can be calculated. Here only the first-order DE is discussed, From Section 3.1, it is known that only the $n_{1}$ will induce the first-order diffracted light, and $\eta_{P, \pm 1}=\left(\tau_{0} \cdot J_{1}\left(k \cdot n_{1} \cdot d\right)\right)^{2}$.

(c) The Calculation of DE of the Mixed Gratings $\left(\eta_{\text {total }}\right)$. The $\eta_{\text {total }}$ can be calculated by submitting the $\eta_{A}$ and $\eta_{P}: \eta_{+1}=$ $\eta_{A,+1}+\eta_{P,+1}$.

(d) The Calculation Results of DE of Fulgide Film in Parallel Polarization Recording. For example, in the parallel linearly polarization recording parallel polarization reading condition $(\vec{C} / / \vec{O} / / \vec{R})$, when $I_{0}=50 \mathrm{~mW} / \mathrm{cm}^{2}$, from the $T_{/ /} \sim$ $E$ curve shown in Figure 6(c), the $T_{/ /}(t) \sim \Delta \varphi$ of the holographic grating at different recording times $t$ can be calculated, from which the $\tau_{/ /}(t) \sim \Delta \varphi$ can be obtained, which is shown in Figure 13. 


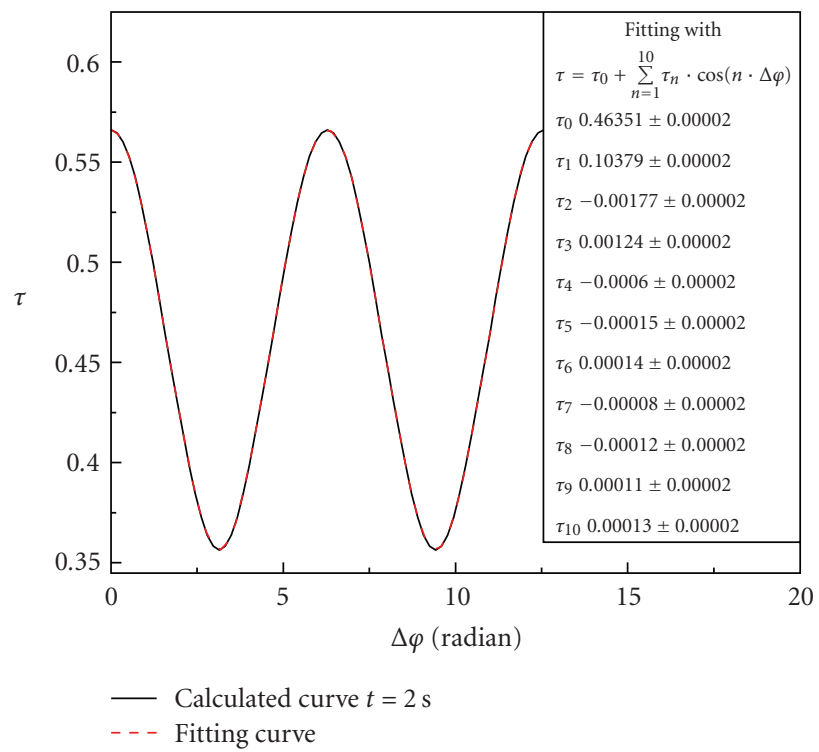

(a)

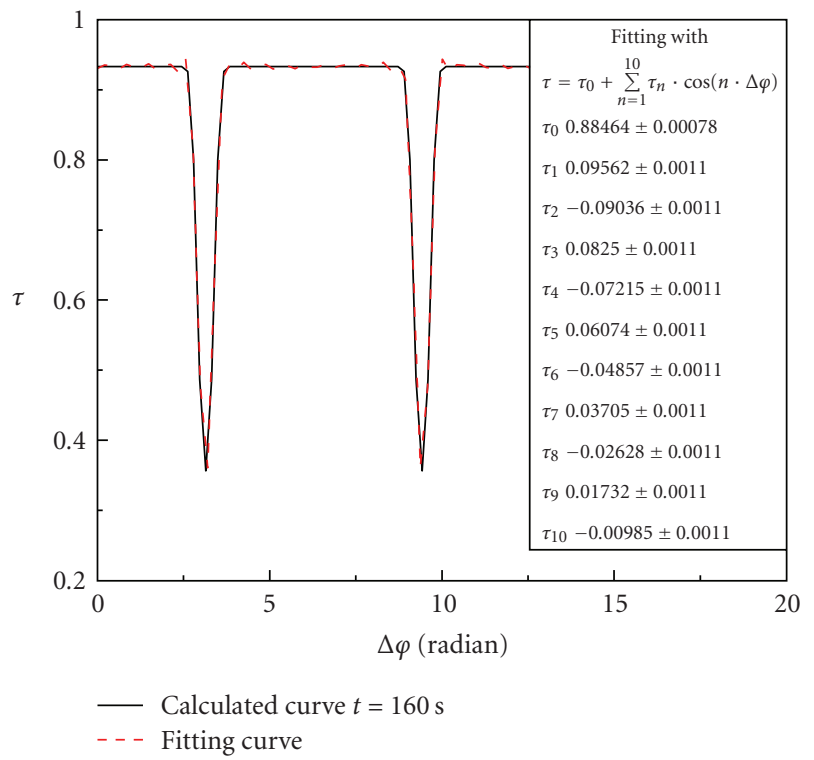

(b)

FIGURE 14: Amplitude transmission grating curves and their simulating curves (a) $t=2 \mathrm{~s}$; (b) $t=160 \mathrm{~s}$.

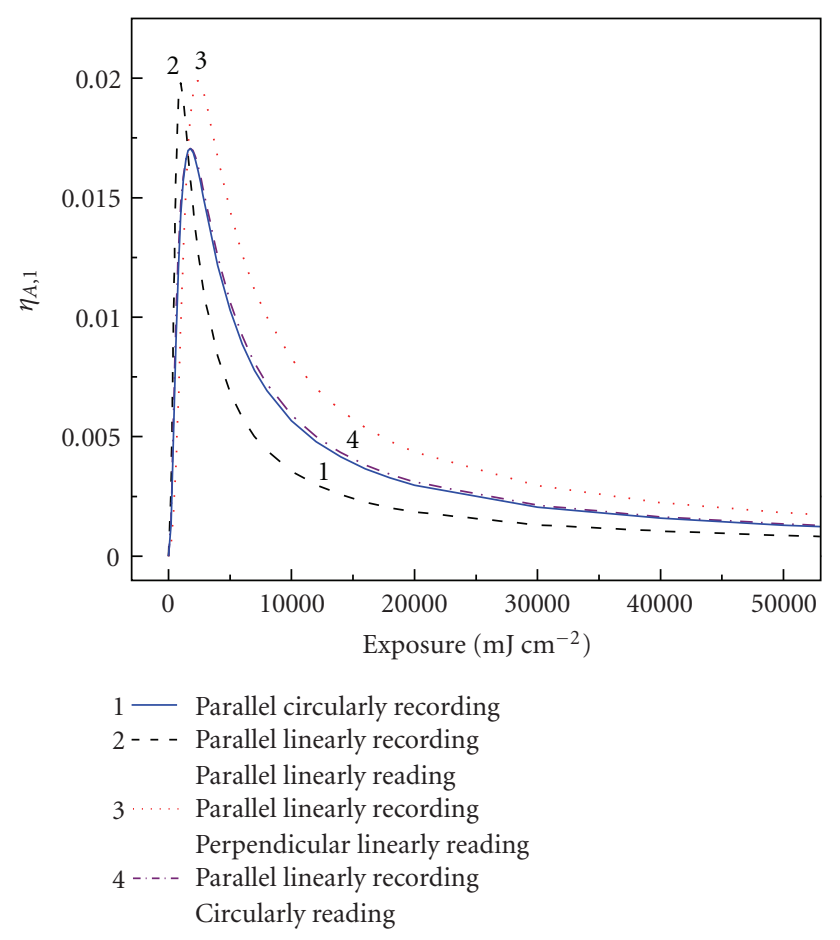

Figure 15: The first-order diffraction efficiencies of the amplitude gratings in parallel polarization holograms.

Every $\tau_{/ /} \sim \Delta \varphi$ curve was spread into Thaler progression, that is was simulated with (30), then the $\tau_{n}(n=0,1, \ldots, 10)$ on the direction of the exiting beam polarization at different $t$ were obtained; for example, in the Figure 14 two typical amplitude transmission grating curves (sine distribution and negative triangle wave function) at different $t$ and their simulated curves are given. So, the $\tau_{n} \sim \bar{E}(n=0,1, \ldots, 10)$ curves can be got, from which the $\eta_{A, \pm n} \sim \bar{E}(n=$ $0,1, \ldots, 10)$ curves can be calculated, from which the firstorder DE $\left(\eta_{A, \pm 1}\right)$ is chosen and shown in Figure 15 as dash line 2. It can be seen that even if the ORR $=1: 1$, because the nonlinear recording the DE will also drop down. The optimal exposure is about $1 \mathrm{~J} / \mathrm{cm}^{2}$, and at the uniformity light recording condition, the maximum $\eta_{A, \pm 1}$ is $2 \%$.

From the $T_{/ /}(t) \sim \Delta \varphi$ curves, the $A_{/ /}(t) \sim \Delta \varphi$ curves can be calculated. After every $A_{/ /}(t) \sim \Delta \varphi$ curve is spread into Thaler progression, the $A_{i}(i=0,1, \ldots, 10)$ on the direction of the exiting beam polarization at different $t$ can be obtained. In Section 2, it is given that $\Delta n(633 \mathrm{~nm}) / \Delta A(633 \mathrm{~nm})=0.00994$. So after the $A_{i}$ multiplexed with 0.00994 , the $n_{i, / /}(i=0,1, \ldots, 10)$ at different $t$ can be got, from which the $\eta_{P, \pm 1} \sim \bar{E}$ can be calculated, which is shown in Figure 16. The DE of the mixed holograph $\eta_{\text {total }}$ can be obtained by submitting that of the amplitude grating and phase grating, shown in Figure 16. It can be seen that at the uniformity light recording condition, the optimal exposure is about $1 \mathrm{~J} / \mathrm{cm}^{2}$, and, the maximum $\eta_{ \pm 1}$ is about $3.56 \%$.

It can be seen that $\eta_{A, \pm 1}$ and $\eta_{P, \pm 1}$ nearly has direct ratio in parallel linearly recording hologrpahy, the decreasing rate of $\eta_{P, \pm 1}$ is a little bit slower than that of $\eta_{A, \pm 1}$, which is ignored in other conditions in parallel polarization recording, and just the $\eta_{A, \pm 1}$ is calculated as before and $\eta_{\text {total }}$ is took as 1.89 times of the $\eta_{A, \pm 1}$.

Using the same method shown before, from $T_{\perp} \sim E$ curve shown in Figure 6(c), the $\eta_{A, \pm n} \sim \bar{E}$ curves for the $\vec{C}$, which has perpendicular polarization to that of the exciting beams $(\vec{C} \perp \vec{O} / / \vec{R})$, are calculated, from which the $\eta_{A, \pm 1}$ is shown in Figure 15. Comparing with the condition of $\vec{C} / / \vec{O} / / \vec{R}$, just the changing rate is slow down. When 
reconstruct with circularly polarized light, the diffracted lights are the superposed light of the diffracted lights of vertical and horizontal polarized light component of the $\vec{C}$, so it will be ellipse polarized lights, whose long axes are along the vertical direction and the $\mathrm{DE}$ are the average values of that of the vertical and horizontal polarized lights, which is shown in Figure 15.

In the parallel circularly polarization holograph (ORR $=1: 1$ ), the $T$ distribution is the same for any kinds of polarization state lights, so the $\mathrm{DE}$ are also the same. Using the same method shown before, from the $T(t) \sim \Delta \varphi$ curves of parallel circularly recording condition shown in (28), the $\eta_{A, \pm n} \sim \bar{E}$ curves of parallel circularly recording holograms are calculated and shown in Figure 15.

(2) The Calculation of DE in the Orthogonal Polarization Recording Condition. From Section 3.1, it is known that in orthogonal polarization recording condition at nonlinear recording area, the DE equations of the linearly recording area shown in Table 1 also can be used. From Figure 6(c), the $\tau_{e} \sim E$ and $\tau_{o} \sim E$ curves of sample under uniformity light irradiation can be calculated. Then from them using the DE formulas, the $\eta_{A,+1} \sim \bar{E}$ curves of the orthogonal linearly polarization holography and orthogonal circularly polarization holography can be calculated, which are shown in Figure 17.

It can be seen that the optimal exposure is $1.2 \mathrm{~J} / \mathrm{cm}^{2}$, and the maximum amplitude grating DE are, respectively, $0.4 \%$ and $1.6 \%$. The decreasing rates are quicker than parallel polarization holography. In Section 2, it is given that in this sample $\Delta n_{B}(633 \mathrm{~nm}) / \Delta A_{D}(633 \mathrm{~nm})=0.006115$. The $\eta_{P,+1}$, the same as the condition in parallel polarization holography, nearly has direct ratio with the $\eta_{A,+1}$, the $\eta_{\text {tatol }}$ in mixing holograph is about 1.28 times of $\eta_{A,+1}$.

3.2.2. The Calculation of $D E \sim \bar{E}$ Curves on the Exposure of the Gaussian Beams. Because in experiment, the writing and reading beams are $633 \mathrm{~nm}$ He-Ne Gaussian beams, so this condition was considered in the calculation below. The light pattern is divided into many intensity homogenous circles, whose intensities and areas are $I_{i}(i=1 \cdots n)$ and $S_{i}(i=$ $1 \cdots n)$, respectively. Using $I_{i}(i=1 \cdots n)$, from the $\eta_{\text {total }} \sim$ $\bar{E}$ curves calculated in Section 3.2.1, the DE of every circle at different time $\eta_{i, t}(i=1 \cdots n)$ can be calculated. When read by the original $\vec{R}$, the diffracted light power $P_{D, t}$ can be got by submitting that of all the circles $P_{D, i, t}=\eta_{i, t} \cdot I_{i} \cdot S_{i}$, and then by dividing by the power of $\vec{C}\left(P_{C}\right)$, the total DE of the sample for the Gaussian reading beam $\eta_{\text {total,Gaussian, } t}=$ $P_{D, t} / P_{C}$ can be obtained. The calculated DE $\sim t$ curves of different kinds of polarization haplographies recorded in this sample, when the writing beams are $633 \mathrm{~nm} \mathrm{He}-\mathrm{Ne}$ Gaussian beams, whose average intensities are $78.6 \mathrm{~mW} / \mathrm{cm}^{2}$ (same with the experiment values) and the original $\vec{R}$ is used as $\vec{C}$, are shown in Figure $9($ b).

It can be seen that the maximum values' ratio of the measured values is basically coincide with the theoretically analyzed one. Only for parallellinearly polarization holo-

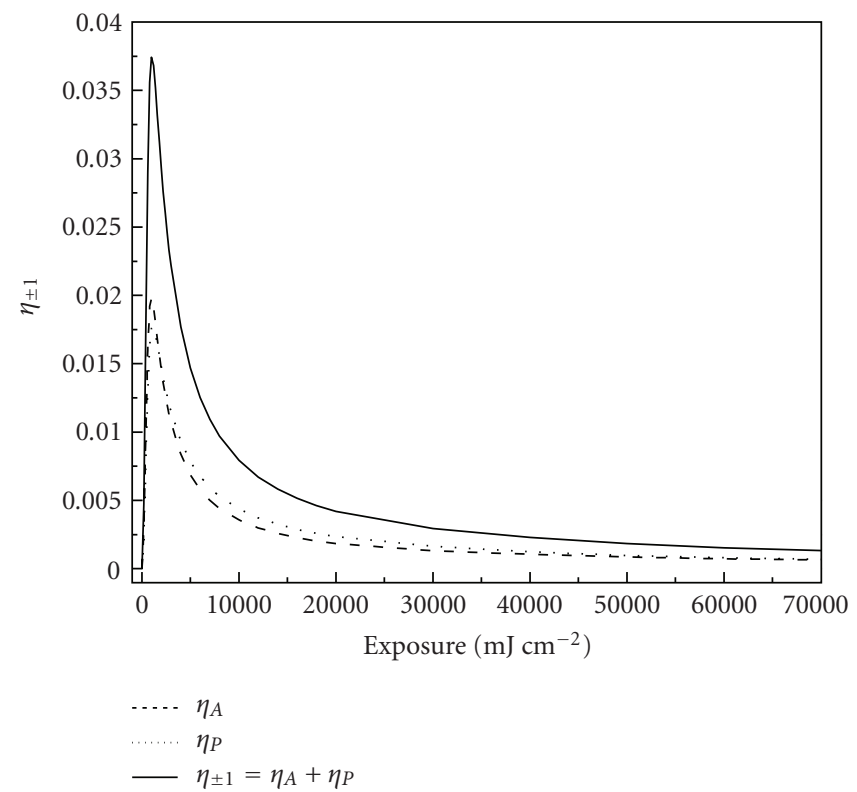

FIgURE 16: The first-order diffraction efficiencies of the amplitude grating and phase grating in the parallel linearly recorded mixing holograph.

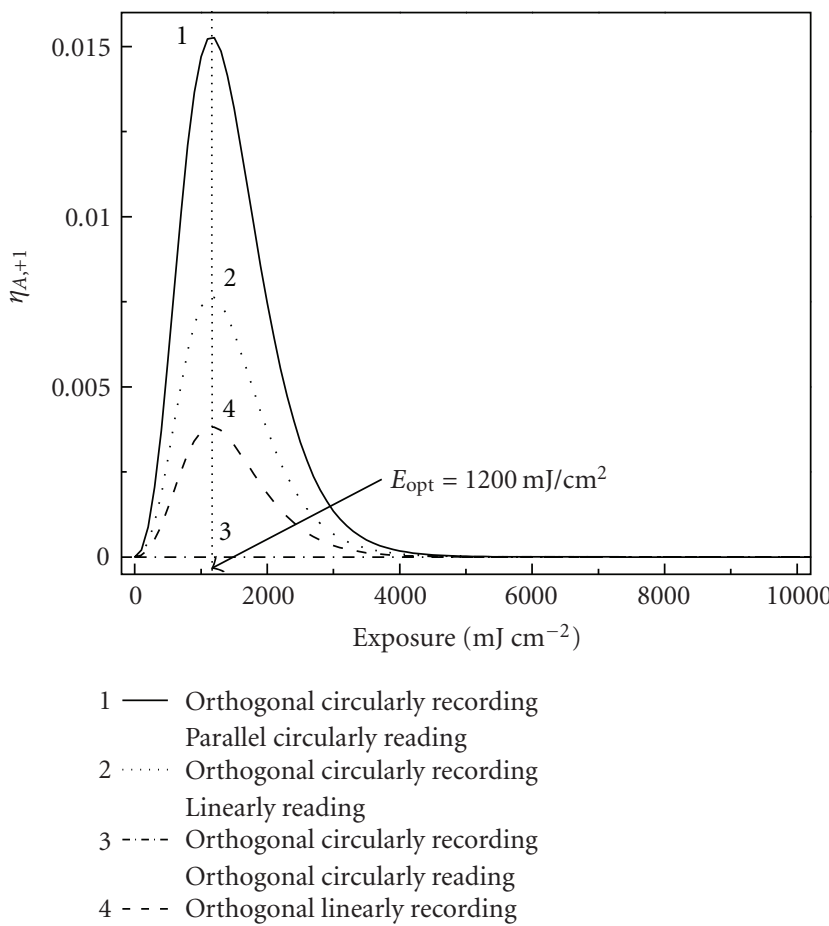

Figure 17: The first-order diffraction efficiencies of the amplitude gratings in orthogonal polarization holograms.

gram, in the orthogonal linearly polarization reconstruction condition $(\vec{C} \perp \vec{O} / / \vec{R})$, the diffraction efficiency is lower than parallel linearly polarization reconstruction condition $(\vec{C} / / \vec{O} / / \vec{R})$. It is because that, in calculation the affection of 


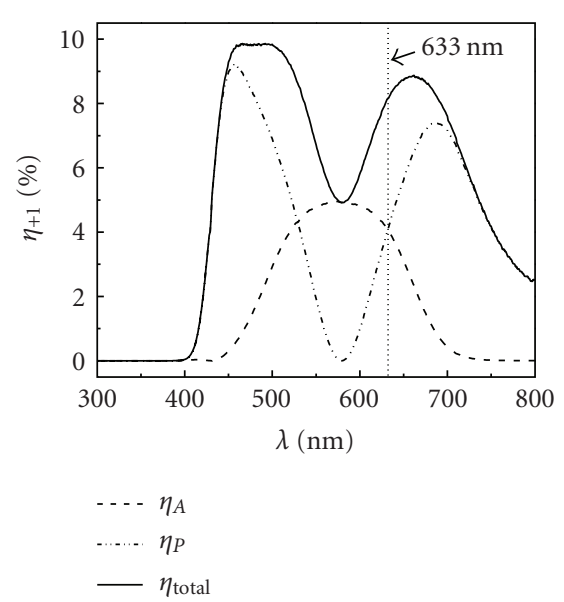

(a)

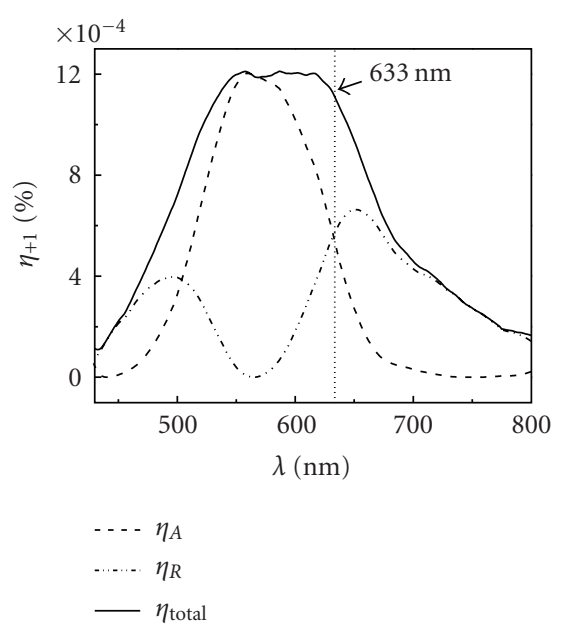

(b)

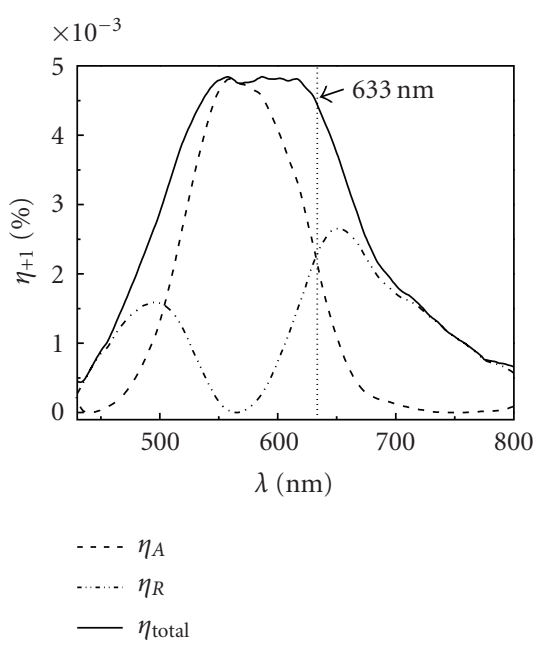

(c)

Figure 18: Diffraction efficiency spectra of the (a) Ordinary holography, (b) Orthogonal linearly polarization holography, and (c) Orthogonal circularly polarization holography.

$\vec{C}$ is not considered, which can be proved to be very small when the $I_{C}=I_{O} / 100=I_{R} / 100$ comparing to the affection of nonlinear absorption of the film, whose detail calculation progress will not be given here, where it also can be deduced that the reading beam affection is larger in $\vec{C} \perp \vec{O} / / \vec{R}$ condition than in $\vec{C} / / \vec{O} / / \vec{R}$ condition.

And the theoretical results are larger than the experimental results, and the reaction is quicker (optimal exposures are about $590 \mathrm{~mJ} / \mathrm{cm}^{2}$ and $430 \mathrm{~mJ} / \mathrm{cm}^{2}$, respectively, in experimental results and theoretical results); this may be caused by: (1) the sample is not homogeneous, the density is different at different area, (2) the incidence angles of beams $\theta$ in the experiment are about $8.2^{\circ}$, so the intensities of them on the sample plane will be $\cos \theta \approx 0.9898$ times of the values used in calculation, (3) the photoreaction rate constants used in calculation is a little bit larger than real ones.

And it can be seen that no matter during the ordinary holograph recording process in photochromic media, or during the polarization holograph recording process in photo-induced anisotropy media, there exits an overshooting peak in the diffraction efficiency, which then decays to a lower permanent level or also to zero. From the theoretical analyses, it can be deduced that it is caused by the diminishing of fringe contrast mainly caused by the nonlinear saturation effects of photoisomerization process and photoinduced anisotropy process. In experiment, there also exit the diminishing of fringe contrast caused by a photochemically active readout beam and unequal intensities of object and reference waves. It can be theoretically calculated that the effects of them show very smaller than that of the nonlinear saturation effects, which will not be given here.

\subsubsection{The DE Spectra of Different Kinds of Holography in} Fulgide Film. Suppose that the holographic recording is a linearity recording, from the spectra of $\Delta A, \Delta n, \Delta A_{D}$, and $\Delta n_{B}$, shown in Figures 3(b) and 4(b), using the DE formulas of different kinds of polarization holographies, shown in Table 1, the DE spectra of the ordinary holograms (i.e., parallel circularly polarization recording) and polarization holograms (orthogonal linearly and orthogonal circularly polarization recording) can be calculated, which are shown in Figure 18, where the solid line, dot dash lines and dash lines indicate the $\eta_{\text {total }}, \eta_{A}$, and $\eta_{P}$, respectively. It can be seen that at $450 \mathrm{~nm}$ and $700 \mathrm{~nm}$ the diffraction efficiencies are higher, while the absorption is very small, where the recorded information can be read out without any photochromic reaction, that is, the nondestructive reconstruction can be realized.

\section{Application in Holographic Image Storage}

4.1. Holographic Image Storage Setup. The property difference of different polarization holograms have be seen in real image holographic storage, whose optical set up is shown in Figure 19. The same as the measurement set up, the $633 \mathrm{~nm}$ He-Ne laser ( $3 \mathrm{~mW}$, random polarized) and $405 \mathrm{~nm}$ diode laser are used as recording and erasing light sources, respectively; shutter $S_{1}$ and $S_{2}$ used to control their exposure times; quarter-wave plates $Q_{1} \sim Q_{3}$ and attenuators $A_{1} \sim A_{2}$ are used to change the polarization states and intensities of the recording (object light $O$ and reference light $R$ ), reading $(C)$ and diffracted $(D)$ waves. We know that in the orthogonal polarization holography, when C-beam has the same polarization state with R-beam, the D-beam has orthogonal polarization with C-beam, while the scattering noise has similar polarization with C-beam. So using the $Q_{3}$ and polarizer $P$ in front of $L_{3}$, the scattering noise can be filtered in orthogonal polarization holography. Located on the front focus plane of Fourier transformation lens $L_{3}$, spatial light modulator (SLM) loads the encoded data image from computer $\mathrm{PC}_{1}$. And the diaphragm $D_{1}$, located on the special frequency spectrum plane of $4 \mathrm{f}$ system composed of $L_{3}$ and $L_{4}$, is used to filter high-order diffractive waves of grating 


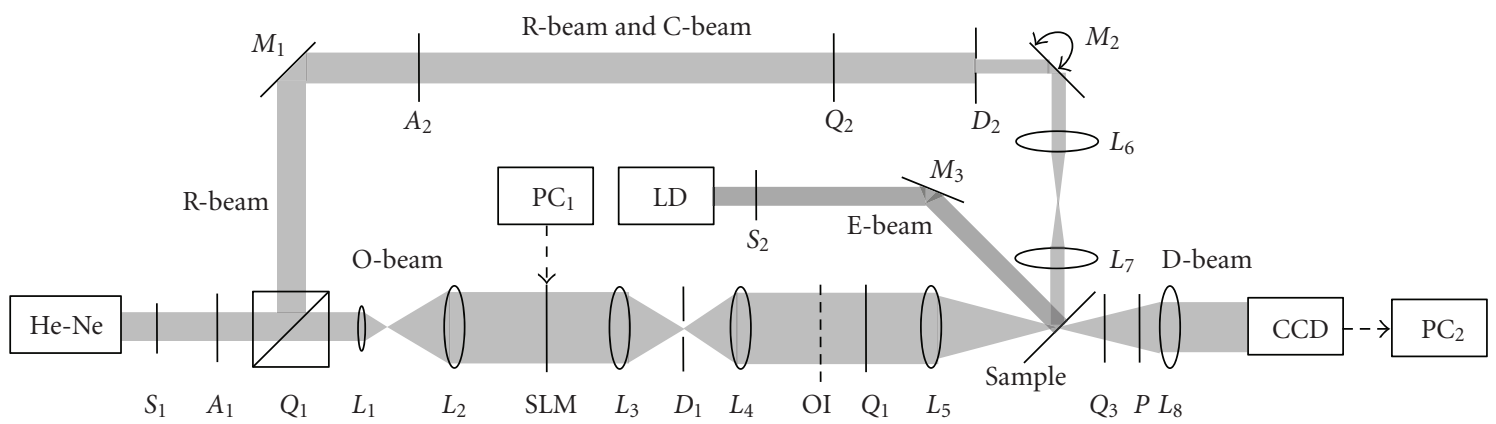

FIGURE 19: Experimental setup of polarization holographic image storage. PBS, polarization beam splitter; $M_{1} \sim M_{4}$, mirrors; $L_{1} \sim L_{8}$, lens; $A_{1}$ and $A_{2}$, continuously adjustable attenuators; $D_{1}$ and $D_{2}$ : diaphragm; $P$, polarizer; $Q_{1} \sim Q_{3}$, quarter-wave plates; $S_{1}$ and $S_{2}$, shutters; LD, $405 \mathrm{~nm}$ laser diode; He-Ne: $633 \mathrm{~nm}$ Helium-Neon laser; O-beam, object beam; R-beam, reference beam; C-beam, reconstruction beam; D-beam, diffracted beam; E-beam, erasure violet light.

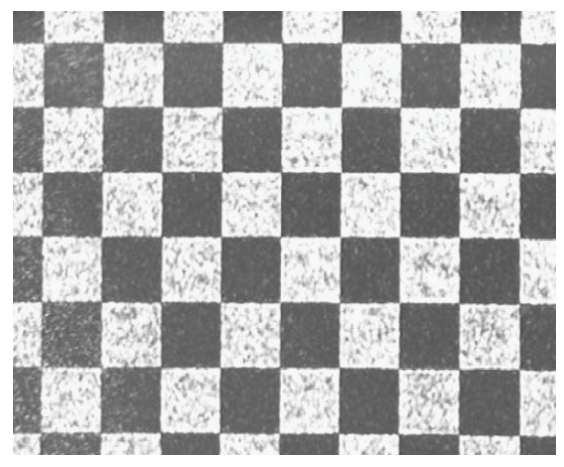

(a)

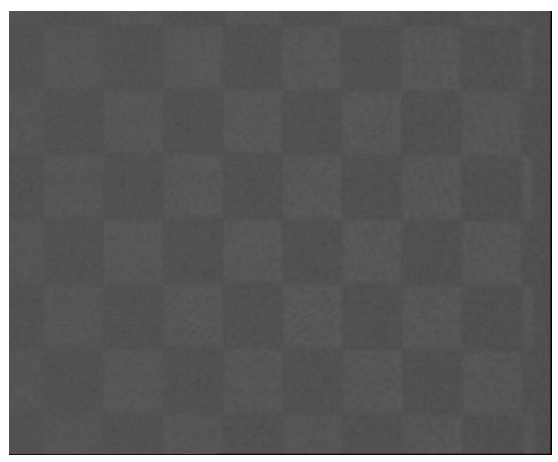

(c)

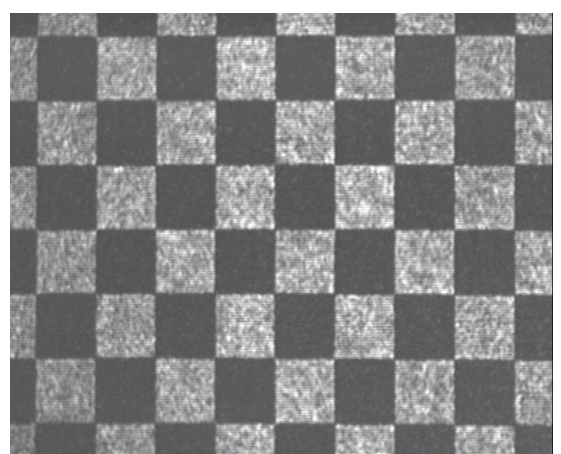

(b)

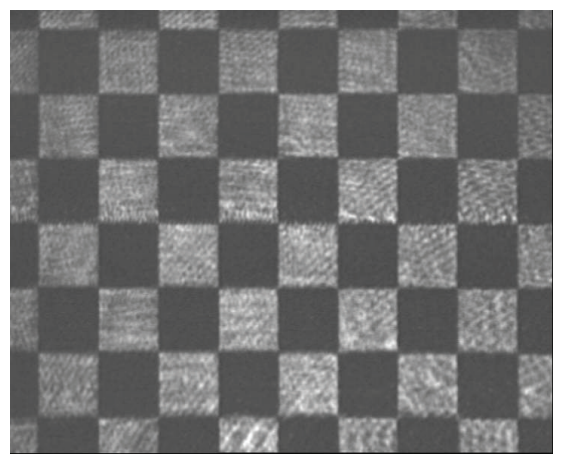

(d)

FIGURE 20: Results of holographic storage experiments with different polarization recording waves on Fulgide film: (a) parallel linear polarization recording; (b) parallel circular polarization recording; (c) orthogonal linear polarization recording; (d) orthogonal circular polarization recording.

structure of SLM, so on the back focus plane of $L_{4}$ the object image (OI) without grating structure can be obtained. In the R-beam path, lens $L_{6}$ and $L_{7}$ comprise another $4 \mathrm{f}$ system, and rotatable mirror $M_{2}$ and the sample, located separately on the focus planes of $L_{6}$ and $L_{7}$, are used to realize angle multiplexing. $L_{5}$ and $L_{8}$ compose another $4 \mathrm{f}$ system, $L_{5}$ performs the Fourier transformation of the object image (OI) at the position of the Fulgide film (sample), and Lens $L_{8}$ performs the inverse Fourier transform at the plane of the CCD camera to yield the diffracted image. Four different polarization recordings like listed in Table 1 are stored with this set up.
4.2. Holographic Image Storage Results. Figure 20 shows the comparison of retrieval diffractive images with different polarization recording waves on the fulgide film, when reconstruction beams have same polarization state with reference beams. It can be seen that these results are same with the measured results shown above. When the intensities of recording wave and readout wave are certain, parallel linear polarization recording obtains the highest diffraction efficiency as well as high scattering noise; parallel circular polarization recording obtains high diffraction efficiency and scattering noise; orthogonal linear polarization recording 


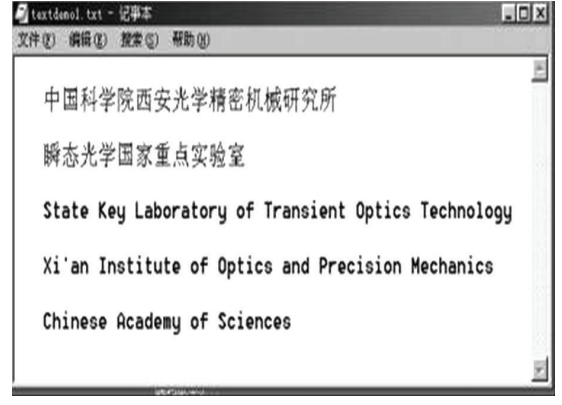

(a)

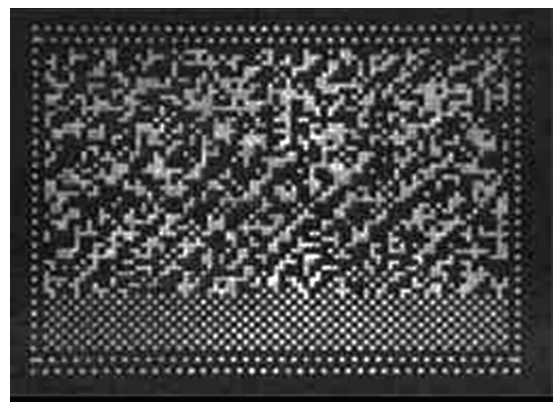

(c)

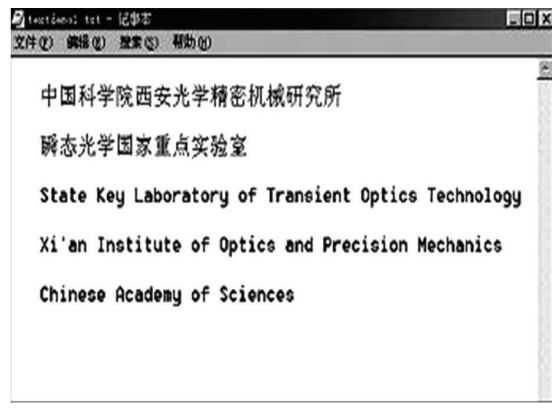

(e)

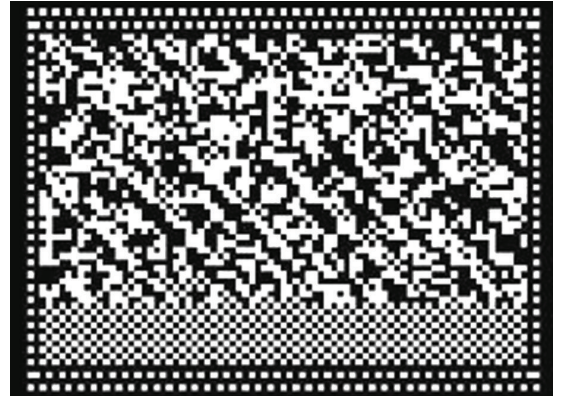

(b)

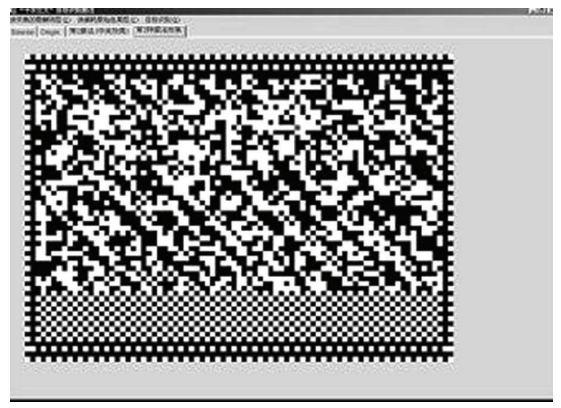

(d)

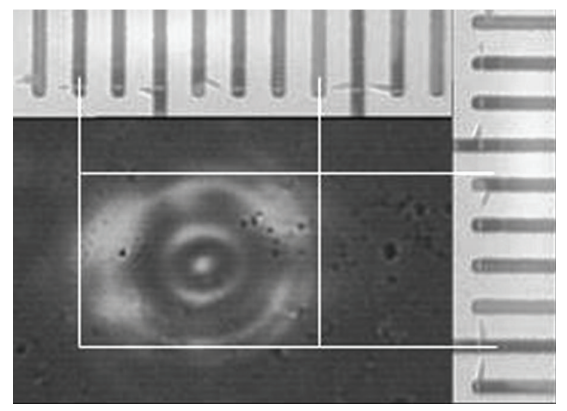

(f)

FIGURE 21: Results of orthogonal circular polarization holographic optical data storage on BR-D96N film by Fourier transformation holographic method. (a) Stored file. (b) Encoded binary monochromic image. (c) Retrieval diffractive image. (d) Decoded result. (e) Retrieved file. (f) Measurement of size of hologram (one grid of the scale corresponds to $10 \mu \mathrm{m}$ ).

obtains low scattering noise and diffraction efficiency; orthogonal circular polarization recording obtains higher diffraction efficiency and high signal-to-noise ratio. So, orthogonal circular polarization recording is chosen as the method of polarization holographic data storage on the fulgide film and Fourier transformation holographic method is chosen to get high storage density.

Figure 21 shows the experimental results of orthogonal circular polarization holographic optical data storage on Fulgide film by Fourier transformation holographic method. The images are separately stored file, encoded binary monochromic image, retrieval diffractive image, decoded result, retrieved file, and measurement of size of holographic image. The retrieval diffractive image that is clear is processed by decoding procedure, and the obtained retrieved file is the same as the stored file. In the experiment, data size of each stored holography is $81 \times 61$ bits, and the size of holography is $60 \mu \mathrm{m} \times 42 \mu \mathrm{m}$. So the storage area density of 2 $\times 10^{8} \mathrm{bits} / \mathrm{cm}^{2}$ is obtained. The nonhomogeneity and flaws of the surface of sample, uncoaxiality of optical elements and uncertainty of adjustment brings out some distortion of the diffractive image and error codes that can be reduced to the minimum by designing reasonable encoding and decoding procedures.

\section{Conclusions}

From the analysis of diffracted wave polarization states in the different kinds of polarization holograms, it can be seen that in the orthogonal polarization holography, if reconstructed with the reference light, the polarization state of the diffracted light is orthogonal to that of the reconstruction light, which is very important to increase the SNR of the holographic storage.

From the dynamic curve calculation of the film, it can be seen that, the decrease of the diffraction efficiencies at 
the high exposure is caused by the nonlinear recording of the sample, and the effect of the reading beam is very small. The maximum values of the diffraction efficiencies at $633 \mathrm{~nm}$ of the parallel linearly, parallel circularly, orthogonal linearly, and orthogonal circularly polarized holograms recorded in 3-indoly-benzylfulgimide/PMMA film are $3.56 \%, 3.026 \%, 0.49 \%$, and $1.96 \%$, respectively. In the condition of the Gaussian beam, the experimentally measured values are smaller than the theoretical calculated ones, but the ratio between the diffraction efficiencies of different kinds of polarization holographs is nearly the same as the calculated ones. The maximum measured diffraction efficiency of the parallel linearly polarization hologram is $1 \%$.

From the diffraction efficiency spectrum, it can be seen that at the wavelengths less than $450 \mathrm{~nm}$ or greater than $700 \mathrm{~nm}$ the nondestructive reading can be realized.

The results obtained in holographic image storage proved that the results obtained in calculation and measurement experiment are correct. In Fourier transformation orthogonal circular polarization recording, high SNR and high density of $2 \times 10^{8} \mathrm{bits} / \mathrm{cm}^{2}$ are obtained.

\section{Acknowledgments}

This research is supported by the Natural Science Foundations of China, under Grant no. 60867001, College Science Foundations of Inner Mongolia, China, under Grant no. NJZZ07007, and the Open Foundations of Key Laboratory of Photochemical Conversion and Optoelectronic Materials, TIPC, CAS, China.

\section{References}

[1] Y. Yokoyama, "Fulgides for memories and switches," Chemical Reviews, vol. 100, no. 5, pp. 1717-1739, 2000.

[2] R. A. Lessard, C. Lafond, F. Ghailane, M. Bolte, A. Tork, and I. Petkov, "Photochromic materials for holographic data storage," in Advanced Photonic Sensors and Applications, vol. 3897 of Proceedings of SPIE, pp. 46-55, December 1999.

[3] R. A. Lessard, A. Tork, C. Lafond, M. Bolte, and A. Ritcey, "Characterization of the dye-doped PMMA, CA and PS films as recording materials," in Organic Photonic Materials and Devices II, vol. 3939 of Proceedings of SPIE, pp. 94-104, January 2000.

[4] C. Lafond, A. Tork, R. A. Lessard, and M. Bolte, "Characterization of the dye-doped PMMA, CA and PS films as recording materials," in Applications of Photonic Technology 4, vol. 4087 of Proceedings of SPIE, pp. 732-740, January 2000.

[5] C. Lafond, O. Pouraghajani, A. Tork, M. Bolte, A. M. Ritcey, and R. A. Lessard, "Characterization of the fulgide-doped PMMA films and investigation of photochromic reaction of Langmuir-Blodgett films as recording materials," in Practical Holography XV and Holographic Materials VII, vol. 4296, pp. 226-236, January 2001.

[6] T. Kardinahl and H. Franke, "Fulgide doped PMMA thin film waveguides for optoelectronics," in Physical Concepts and Materials for Novel Optoelectronic Device Applications II, vol. 1985 of Proceedings of SPIE, pp. 742-751, 1993.
[7] T. Kardinahl and H. Franke, "Gratings in nonlinear optical polymers for integrated optical device applications," in Nonlinear Optical Properties of Organic Materials VI, vol. 2025 of Proceedings of SPIE, pp. 574-578, 1993.

[8] T. Kardinahl and H. Franke, "Photoinduced refractive-index changes in fulgide-doped PMMA films," Applied Physics A Materials Science \& Processing, vol. 61, no. 1, pp. 23-27, 1995.

[9] T. Kardinahl and H. Franke, "Nonlinear optical gratings in organic photochromic films," in Linear and Nonlinear Integrated Optics, vol. 2212 of Proceedings of SPIE, pp. 346353, 1994.

[10] N. Menke, B. Yao, Y. Wang et al., "Optical image processing using the photoinduced anisotropy of pyrryfulgide," Journal of the Optical Society of America A, vol. 23, no. 2, pp. 267-271, 2006.

[11] Y. Chen, C. Wang, M. Fan, B. Yao, and N. Menke, "Photochromic fulgide for holographic recording," Optical Materials, vol. 26, no. 1, pp. 75-77, 2004.

[12] N. Menke, B. Yao, Y. Wang et al., "Spectral relationship of photoinduced refractive index and absorption changes in fulgide films," Journal of Modern Optics, vol. 55, no. 6, pp. 1003-1011, 2008. 

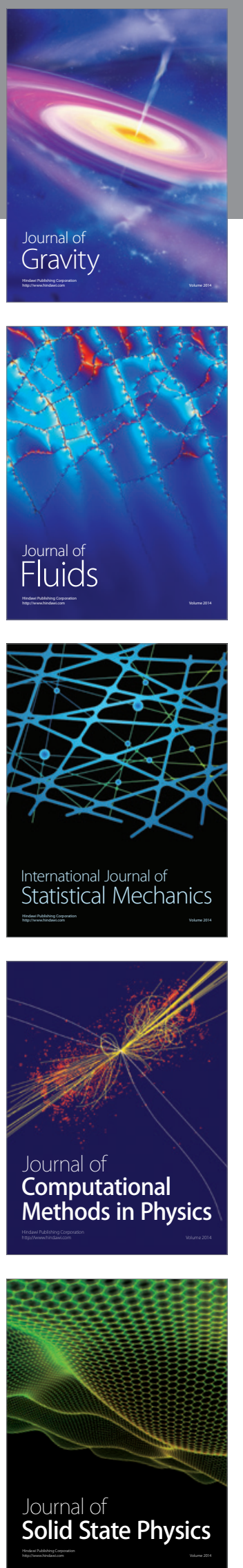

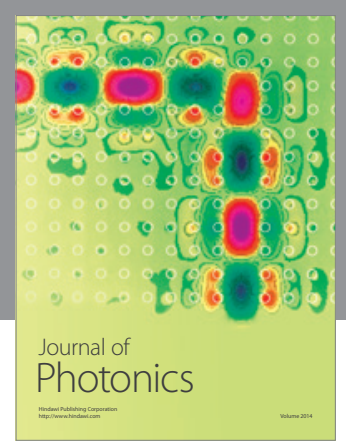

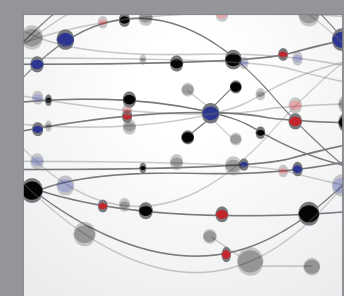

The Scientific World Journal
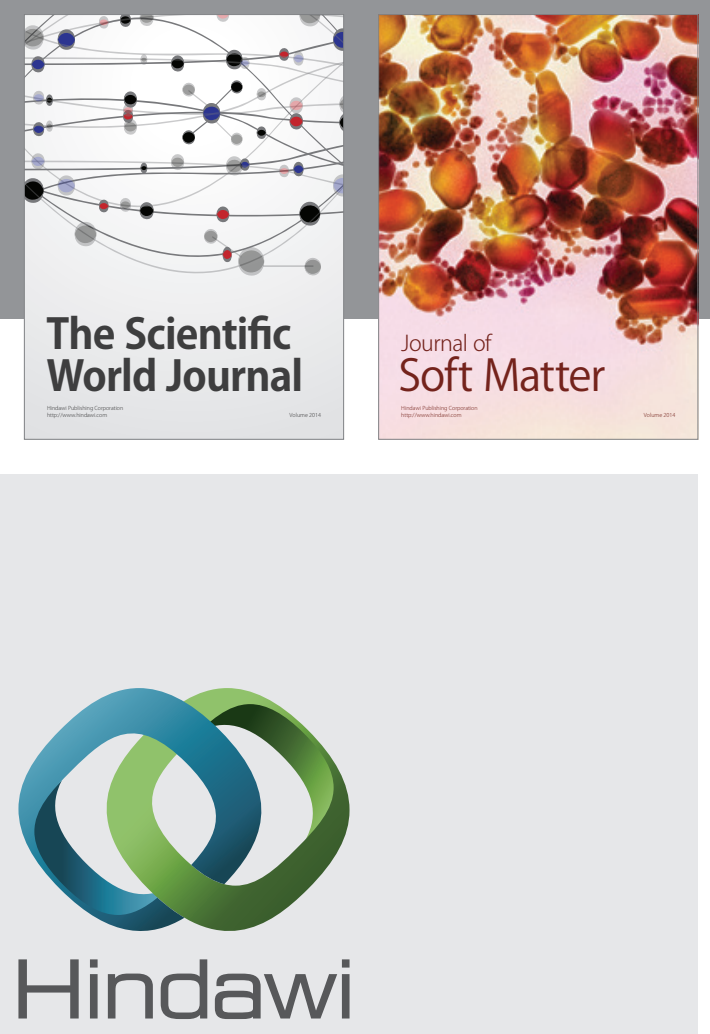

Submit your manuscripts at

http://www.hindawi.com
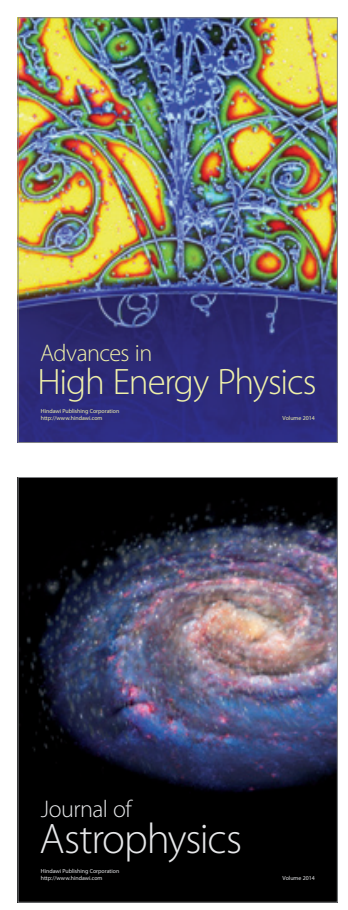
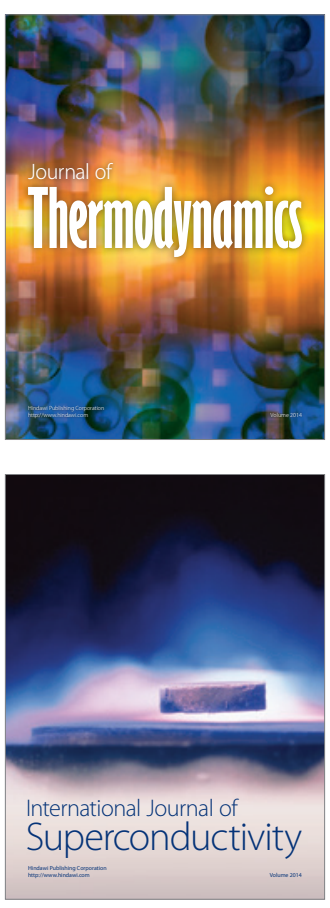
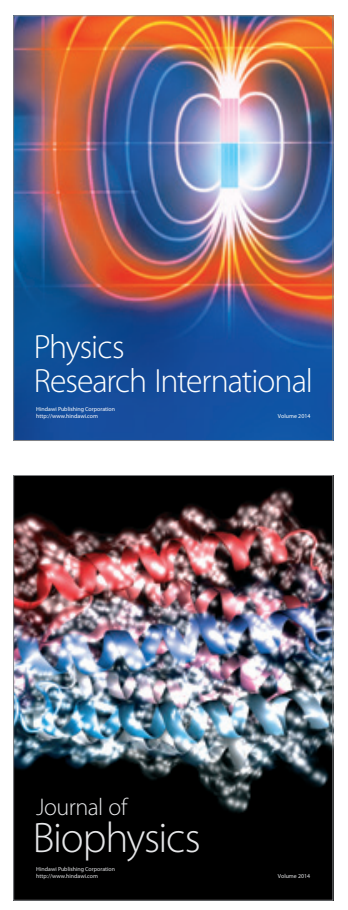
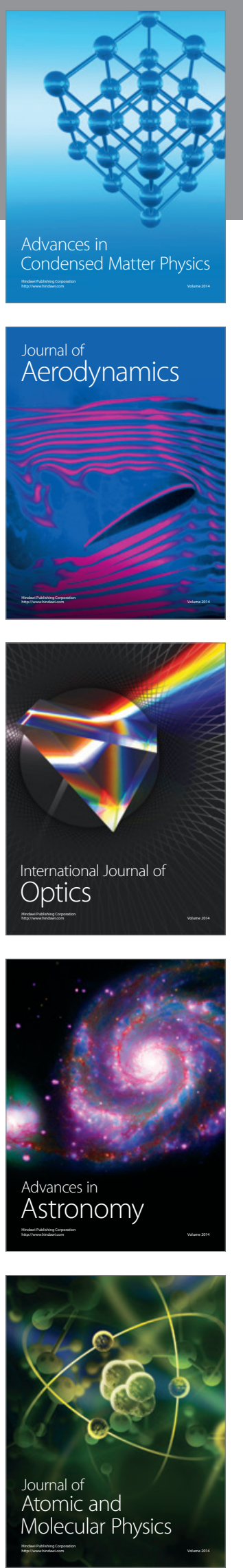